\title{
Article \\ A Theoretical Analysis of a Fractional Multi-Dimensional System of Boundary Value Problems on the Methylpropane Graph via Fixed Point Technique
}

\author{
Shahram Rezapour ${ }^{1,2,+}\left(\mathbb{D}\right.$, Chernet Tuge Deressa ${ }^{3,+}$, Azhar Hussain ${ }^{4, *,+}+\mathbb{C}$, Sina Etemad ${ }^{1,+}+\mathbb{D}$ and Reny George ${ }^{5,6, *,+}$ \\ and Bashir Ahmad ${ }^{7,+}$ (D) \\ 1 Department of Mathematics, Azarbaijan Shahid Madani University, Tabriz 751-71379, Iran; \\ sh.rezapour@azaruniv.ac.ir (S.R.); sina.etemad@azaruniv.ac.ir (S.E.) \\ 2 Department of Medical Research, China Medical University Hospital, China Medical University, \\ Taichung 40402, Taiwan \\ 3 Department of Mathematics, College of Natural Sciences, Jimma University, Jimma, Ethiopia; \\ chernet.deressa@ju.edu.et \\ 4 Department of Mathematics, University of Sargodha, Sargodha 40100, Pakistan \\ 5 Department of Mathematics, College of Science and Humanities in AlKharj, \\ Prince Sattam Bin Abdulaziz University, Al-Kharj 11942, Saudi Arabia \\ 6 Department of Mathematics and Computer Science, St. Thomas College, Bhilai 490006, India \\ 7 Nonlinear Analysis and Applied Mathematics (NAAM)-Research Group, Department of Mathematics, \\ Faculty of Science, King Abdulaziz University, P.O. Box 80203, Jeddah 21589, Saudi Arabia; \\ bashir_qau@yahoo.com \\ * Correspondence: azhar.hussain@uos.edu.pk (A.H.); r.kunnelchacko@psau.edu.sa (R.G.) \\ + These authors contributed equally to this work.
}

check for updates

Citation: Rezapour, S.; Deressa, C.T.; Hussain, A.; Etemad, S.; George, R.; Ahmad, B. A Theoretical Analysis of a Fractional Multi-Dimensional System of Boundary Value Problems on the Methylpropane Graph via Fixed Point Technique. Mathematics 2022, 10, 568. https://doi.org/ $10.3390 /$ math10040568

Academic Editor: Vasily E. Tarasov

Received: 3 January 2022

Accepted: 9 February 2022

Published: 12 February 2022

Publisher's Note: MDPI stays neutral with regard to jurisdictional claims in published maps and institutional affiliations.

Copyright: (C) 2022 by the authors. Licensee MDPI, Basel, Switzerland. This article is an open access article distributed under the terms and conditions of the Creative Commons Attribution (CC BY) license (https:// creativecommons.org/licenses/by/ $4.0 /)$.

\begin{abstract}
Few studies have investigated the existence and uniqueness of solutions for fractional differential equations on star graphs until now. The published papers on the topic are based on the assumption of existence of one junction node and some boundary nodes as the origin on a star graph. These structures are special cases and do not cover more general non-star graph structures. In this paper, we state a labeling method for graph vertices, and then we prove the existence results for solutions to a new family of fractional boundary value problems (FBVPs) on the methylpropane graph. We design the chemical compound of the methylpropane graph with vertices specified by 0 or 1 , and on every edge of the graph, we consider fractional differential equations. We prove the existence of solutions for the proposed FBVPs by means of the Krasnoselskii's and Scheafer's fixed point theorems, and further, we study the Ulam-Hyers type stability for the given multi-dimensional system. Finally, we provide an illustrative example to examine our results.
\end{abstract}

Keywords: fractional differential equation; boundary value problem; methylpropane graph; the Caputo fractional derivative; stability

\section{Introduction}

Some natural phenomena throughout the world have been studied using initial and BVPs for many years. Due to the broad diversity of BVPs, many academics have turned to mathematical tools and computer simulation software to explore a variety of practical processes. It has recently been shown that fractional differential equations may be used to describe many diverse applications in the applied sciences (see for examples, [1-10]). One of our purposes in this work is to extend the theoretical aspects of some applied concepts in chemistry to increase our abilities to model some processes in chemical reactions. Once that is accomplished, computer engineers will be able to build software that allow everyone to conduct chemical experiments without utilizing real materials. This will allow for the most effective management of the environmental resources that are available. We may utilize fractional calculus for this, which is useful in similar situations. It is possible to solve 
fractional differential-equation-based mathematical models, derive the solution functions, then examine the qualitative behavior of solutions under specific boundary constraints using certain approaches. As may be shown in several published publications, some problems have been addressed before (see for examples, [11-23]).

Water pipes, gas transmission lines, population increase and computer network expansion are only a few examples of industrial and medical areas where new applied models have been developed to analyze the processes devised by engineers in these disciplines. There has been some interest in studying mathematical models that are expressed on graphs via ordinary or fractional differential equations, due to their graph representation. In fact, on a graph, BVPs are specified on every edge as a set of differential equations, along with particular boundary conditions at every vertex of the graph.

In 1980, Lumer's work on differential equations defined on graphs is the preliminary stage for this theory [24]. The local operators established on ramification spaces were used to study generic evolution equations. Nicaise explored the propagation of nerve impulses using a similar system [25]. With respect to a geometric graph with linear differential equations, Zavgorodnij investigated those solutions that were coordinated on their inner vertices in 1989 [26]. An adjoint boundary problem was formulated by him, and the adjointness criteria were established [26]. On the basis of graph representation, Gordeziani et al. have studied the uniqueness-existence of solutions to ODEs in 2008 [27] and solved the given BVP using the double sweep method and provided a numerical methodology.

In the majority of the referenced studies, differential equations were evaluated on a graph and their solutions were determined using numerical as well as computational techniques. The existence aspects of solutions to fractional BVPs on graphs is, however, only established in a few publications using approaches from fixed point theory [28,29].

Graef et al. were the first researchers who used fixed point theory to explore the existing results [28]. They presented a three-vertex-star graph $G=\mathcal{V}(\mathbb{G}) \cup \mathcal{E}(\mathbb{G})$, in which $\mathcal{V}(\mathbb{G})=\left\{\mathrm{p}_{0}, \mathrm{p}_{1}, \mathrm{p}_{2}\right\}$ and $\mathcal{E}(\mathbb{G})=\left\{\mathrm{e}_{1}=\overrightarrow{\mathrm{p}_{1} \mathrm{p}_{0}}, \mathrm{e}_{2}=\overrightarrow{\mathrm{p}_{2} \mathrm{p}_{0}}\right\}$, the vertex $\mathrm{p}_{0}$ regarded as junction node, the edge $e_{i}=\overrightarrow{p_{i} p_{0}}$ connecting the vertices $p_{i}$ to $p_{0}$ having length $l_{i}=\left|\overrightarrow{p_{i} p_{0}}\right|$ for $i=1,2$ (Figure 1 ). At every $\mathrm{e}_{\mathrm{i}}=\overrightarrow{\mathrm{p}_{\mathrm{i}} \mathrm{p}_{0}}$, by considering the origin at boundary vertices $p_{1}$ and $p_{2}$, a local coordinate system is made together with the coordinate $t \in\left(0, l_{\mathrm{i}}\right)$. A nonlinear fractional differential equation system was developed by Graef et al. on each edge $\mathrm{e}_{\mathrm{i}}=\overrightarrow{\mathrm{p}_{\mathrm{i}} \mathrm{p}_{0}}$ as follows $(i=1,2)$

$$
-\mathcal{D}_{0}^{\alpha} \mathrm{y}_{\mathrm{i}}(\mathrm{t})=\mathrm{g}_{\mathrm{i}}(\mathrm{t}) \mathrm{h}_{\mathrm{i}}\left(\mathrm{t}, \mathrm{y}_{\mathrm{i}}(\mathrm{t})\right),
$$

in which, $\mathrm{t} \in\left(0, \mathrm{l}_{\mathrm{i}}\right)$ via the $\mathrm{BCs}$

$$
\mathrm{y}_{1}(0)=\mathrm{y}_{2}(0)=0, \mathrm{y}_{1}\left(\mathrm{l}_{1}\right)=\mathrm{y}_{2}\left(\mathrm{l}_{2}\right), \quad \mathcal{D}_{0}^{\beta} y_{1}\left(\mathrm{l}_{1}\right)+\mathcal{D}_{0}^{\beta} y_{2}\left(\mathrm{l}_{2}\right)=0,
$$

where $\beta \in(0, \alpha), \alpha \in(1,2], g_{i} \in C\left(\left[0, l_{i}\right], \mathbb{R}\right)$ and $h_{i} \in C\left(\left[0, l_{i}\right] \times \mathbb{R}, \mathbb{R}\right)$ with $g_{i}(t) \neq 0$ on $\left[0,1_{i}\right], \mathcal{D}_{0}^{\alpha}$ and $\mathcal{D}_{0}^{\beta}$ regarded as RL-fractional derivatives. Their desired results are obtained by applying Schauder's theorem and the contraction principle.

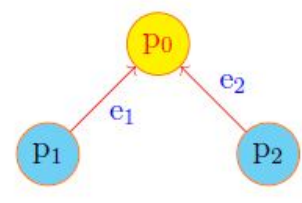

Figure 1. Star graph $\mathbb{G}$ having two edges.

Mehandiratta et al. in 2019 [29] presented an extension of star graph having $n$ edges and $n+1$ vertices. Authors made a generic star graph $\mathbb{G}$ that including $\mathcal{V}(\mathbb{G})=$ $\left\{\mathrm{p}_{0}, \mathrm{p}_{1}, \ldots \mathrm{p}_{\mathrm{n}}\right\}$ and $\mathcal{E}(\mathbb{G})=\left\{\mathrm{e}_{1}=\overrightarrow{\mathrm{p}_{1} \mathrm{p}_{0}}, \mathrm{e}_{2}=\overrightarrow{\mathrm{p}_{2} \mathrm{p}_{0}}, \ldots, \mathrm{e}_{\mathrm{n}}=\overrightarrow{\mathrm{p}_{\mathrm{n}} \mathrm{p}_{0}}\right\}$, in which $\mathrm{l}_{\mathrm{i}}=\left|\overrightarrow{\mathrm{p}_{\mathrm{i}} \mathrm{p}_{0}}\right|$ is considered as the length of $\mathrm{e}_{\mathrm{i}}$ joining vertices $\mathrm{p}_{\mathrm{i}}$ to $\mathrm{p}_{0}\left(i \in \mathbb{N}_{1}^{n}\right)$. 
The following system consists of the BVPs that Mehandiratta et al. defined them on the edges of the above graph $\mathbb{G}$ as

$$
\begin{cases}{ }^{c} \mathcal{D}_{0}^{\alpha} y_{i}(t)=f_{i}\left(t, y_{i}(t),{ }^{c} \mathcal{D}_{0}^{\beta} y_{i}(t)\right), & \left(t \in\left(0, l_{i}\right)\right), \\ y_{i}(0)=0, & y_{i}\left(l_{i}\right)=y_{k}\left(l_{k}\right)(i \neq k), \quad \sum_{i=1}^{n} y_{i}^{\prime}\left(l_{i}\right)=0\end{cases}
$$

in which $0<\beta \leq \alpha-1, \alpha \in(1,2], f_{i} \in C\left(\left[0,1_{i}\right] \times \mathbb{R}^{2}, \mathbb{R}\right)$, and ${ }^{c} \mathcal{D}_{0}^{\gamma}$ is the $\gamma^{\text {th }}$-Caputo derivative. The authors presented the transforms $\mathrm{v}=\frac{\mathrm{t}}{\mathrm{l}_{\mathrm{i}}} \in[0,1]$ and $r(v)=y(t)=y\left(l_{i} v\right)$ for $\mathrm{t} \in\left[0, \mathrm{l}_{\mathrm{i}}\right]$, and showed that the equality ${ }^{c} \mathcal{D}_{0}^{\alpha} y(t)=l_{i}^{-\alpha}\left({ }^{c} \mathcal{D}_{0}^{\alpha} r(v)\right)$ holds. Next, they transformed the system of FBVPs on the graph (1) to the succeeding system of FBVPs over the interval $[0,1]$ by performing these transformations

$$
{ }^{c} \mathcal{D}_{0}^{\alpha} r_{i}(v)=l_{i}^{\alpha} h_{i}\left(v, r_{i}(v), l_{i}^{-\beta^{c}} \mathcal{D}_{0}^{\beta} r_{i}(v)\right), \quad(v \in[0,1])
$$

with $r_{i}(0)=0, r_{i}(1)=r_{k}(1)$ for $i \neq k$ and $\sum_{i=1}^{n} l_{i}^{-1} r_{i}^{\prime}(1)=0$, with $r_{i}(v)=u_{i}\left(l_{i} v\right)$ and $\mathrm{h}_{\mathrm{i}}(\mathrm{v}, \mathrm{y}, \mathrm{w})=\mathrm{f}_{\mathrm{i}}\left(\mathrm{l}_{\mathrm{i}} \mathrm{v}, \mathrm{y}, \mathrm{w}\right)$ for $\mathrm{i} \in \mathbb{N}_{1}^{\mathrm{n}}$.

These works have inspired us to expand their BVPs to a novel BVP on the methylpropane graph. In fact, it is a generalized graph compared to star graphs. In the future, new scholars may generalize these notions to different graphs as new ideas.

\section{Preliminaries}

The Graph in the context of methylpropane compound is introduced in this section for defining a new category of fractional BVPs on it. Let us first bring the attention of readers to two crucial aspects regarding the methodologies employed in $[28,29]$.

(1) As shown in Figures 1 and 2, the authors of both articles assume that $\mathbb{G}$ is a star graph with one junction node $p_{0}$, but in general, graph $G$ may not be a star graph and may have more than one junction node. Figure 3 shows five junction nodes, as an example.

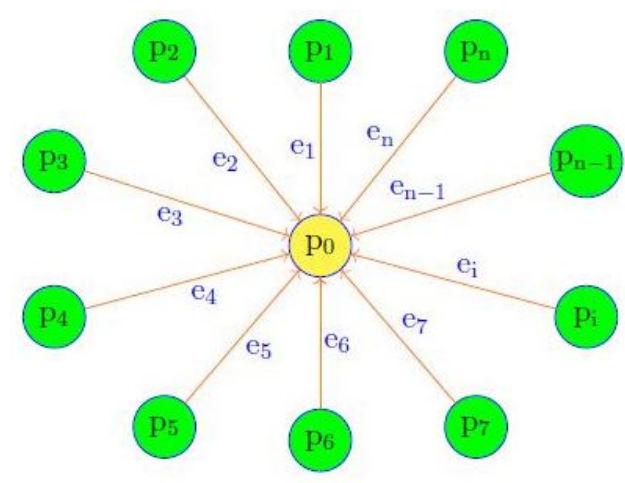

Figure 2. Star graph $\mathbb{G}$ having $n$ edges.

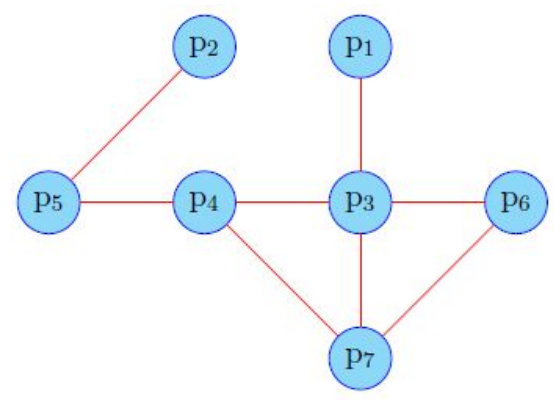

Figure 3. A non-star graph $\mathbb{G}$ with five junction nodes. 
(2) It is important to note that both articles treat the length of every edge as a variable $\left|e_{i}\right|=1_{i}$ for $i \in\{1,2, \ldots, n\}$, where $n$ represents how many vertices there are in the graph $\mathbb{G}$. Then, for the normalization of all edges, they use change of variable and transform $\left[0, l_{i}\right]$ to interval $[0,1]$, whereas in the beginning, one might assume that all edges are the same length $\left|e_{i}\right|=1$ without determining boundary vertex as the origin on each edge. A novel approach for labeling the vertices is proposed as a means of achieving this goal. This allows us to label each vertex of the graph with either 0 or 1 . To put it as another way, each vertex has a different name (label) based on the direction of related edge. A vertex's label and its ending vertex's label are treated as 0 and 1, respectively, when we move along an edge arbitrarily. Hence some labels are 0 , some are 1 , some are both 0 and 1 and each origin in every edge is not fixed; it is changed with each change in direction of the corresponding edge. It is not necessary to normalize the length of all edges utilizing the mentioned transforms; therefore, we are permitted to select one of the two vertices of each edge as its origin. Figure 4 shows an example of how labeling may be used in this situation. We begin to walk along the edges of this graph in the first step from the blue vertex.

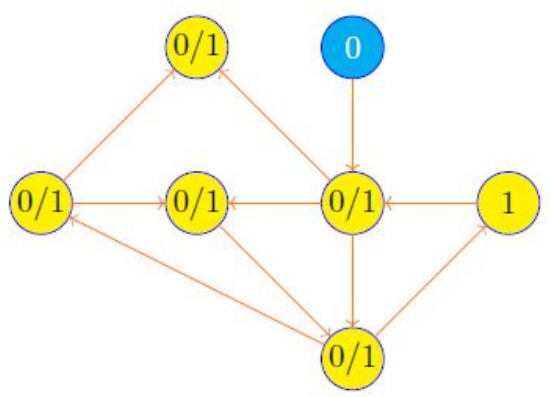

Figure 4. A general graph $\mathbb{G}$ with labels 0 or 1.

In this paper, on the methylpropane graph, we investigate several desirable existence theorems for solutions of a new class of FBVPs (Figure 5).

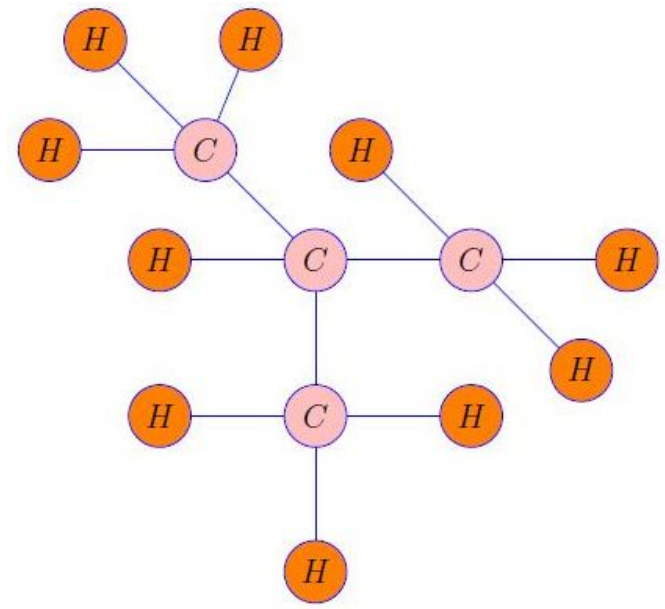

Figure 5. A sketch of methylpropane $\mathrm{HC}\left(\mathrm{CH}_{3}\right)_{3}$ in the graph settings.

The chemical compound of methylpropane or isobutane has the molecular formula $\mathrm{HC}\left(\mathrm{CH}_{3}\right)_{3}$, where $(\mathrm{H})$ and $(\mathrm{C})$ are hydrogen and carbon. Methylpropane is an isomer of Butane, which is used as a precursor molecule in the petrochemical industry. This compound consists of three carbon-carbon bonds and three sub-branches $\mathrm{CH}_{3}$. Inspired by this structure, Hydrogen atoms and carbon atoms are regarded as the graph' vertices. Graph's edges stand for the chemical bonds that occur between atoms. This molecular graph is not a star graph, thus the technique applied in $[28,29]$ which assigns the origin at boundary nodes except the junction node $\mathrm{p}_{0}$, will not work here because there exist more 
than one junction node on this graph (four junction nodes). Consequently, we must adopt a new approach. We may label the vertices of this graph with either 0 or 1 , and we can regard unit value $l_{j}=1$ as the length of each edge (see Figure 6 ).

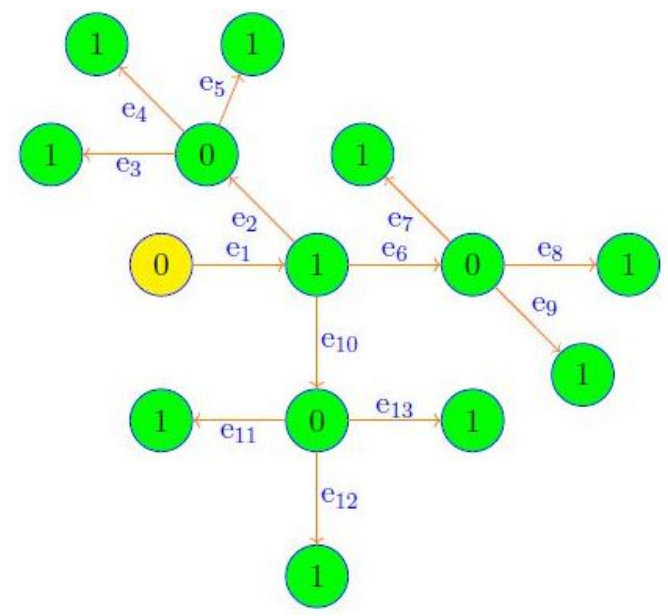

Figure 6. The graph of $\mathrm{HC}\left(\mathrm{CH}_{3}\right)_{3}$ with labels 0 or 1 .

The existence of solutions in relation to the multi-dimensional nonlinear multi-term FBVPs are studied in this manuscript by defining the graph of methylpropane labeled with 0 or 1 , as shown above:

$$
\left\{\begin{array}{l}
{ }^{c} \mathcal{D}_{0}^{\vartheta} z_{j}(t)=h_{j}\left(t, z_{j}(t), z_{j}^{\prime}(t)\right)+k_{j}\left(t, z_{j}(t), z_{j}^{\prime}(t)\right), \quad(t \in[0,1]) \\
z_{j}(0)+{ }^{c} \mathcal{D}_{0}^{\beta} z_{j}(0)+z_{j}^{\prime}(1)=0, \\
\int_{0}^{1}\left[z_{j}(\xi)+{ }^{c} \mathcal{D}_{0}^{\beta} z_{j}(\xi)+z_{j}^{\prime}(\xi)\right] \mathrm{d} \xi=0
\end{array}\right.
$$

where $\vartheta \in(1,2), \beta \in(0,1), h_{j}, k_{j} \in C\left([0,1] \times \mathbb{R}^{2}, \mathbb{R}\right)$ for $j \in \mathbb{N}_{1}^{13}:=\{1,2, \ldots, 13\}$, in which $n=13$ stands for the number of edges of methylpropane graph s.t. $\left|\mathrm{e}_{\mathbf{j}}\right|=1$, and ${ }^{c} \mathcal{D}_{0}^{\vartheta}$ regards the $\vartheta^{t h}$-Caputo derivative. Here in the boundary conditions, we consider the values of the unknown functions $z_{j}$, and their $\beta^{\text {th }}$-derivatives $\left({ }^{c} \mathcal{D}_{0}^{\beta} z_{j}\right)$ and their first-order derivatives $\left(z_{j}^{\prime}\right)$ in the terminal points, and also we consider the integral of sum of them in the mid-points of the time domain $[0,1]$.

Regarding the relationship between the above system of FBVPs and the molecular graph of methylpropane, it should be noted that the main reason for this study is the growing importance of chemical graph theory. This field of mathematics relates graph theory to chemistry, and investigates the chemical changes resulting from interatomic bonds along bond lines and their effects, and these studies are performed in the form of various models of fractional differential equations. Instances of these new applications can be found in bio-macromolecules and chemical kinetics. As the main idea of this work, the results of the given fractional BVPs (2) about the existence of solutions can be interpreted in various meanings in relation to organic chemistry. As a result, any solution $z_{j}(t)$ at every edge $e_{j}$ might represents the bond polarity, bond strength, bond energy, etc. Moreover, the integer and fractional order derivatives of the unknown functions $z_{j}$, i.e., $z_{j}^{\prime}(t)$ and ${ }^{c} \mathcal{D}_{0}^{\beta} z_{j}(t)$ can interpret some chemical notions in this direction. Because ${ }^{c} \mathcal{D}_{0}^{\beta} z_{j}(t)$ has a nonlocal nature, it can show some useful properties of the curves of solutions over a large extent of a time interval $[0,1]$ during a chemical interaction, while $z_{j}^{\prime}(t)$ has a local nature and it can interprets the velocity of the same chemical interactions at a specific time $t \in[0,1]$. Further, 
$h_{j}$ and $k_{j}$ are defined as functions of these quantities with respect to the time $t$ on each edge $e_{j}, j \in \mathbb{N}_{1}^{13}$.

On the other hand, as we said above, $n=13$ shows the number of edges of the methylpropane graph and this index can be changed by increasing or decreasing the number of edges in different molecular structures. In other words, we can generalize this number to each arbitrary value $k \in \mathbb{N}$ and this change will not affect the computational disorder. We are sure that in the future, the theory of chemical reactions and also chemical graph theory may benefit from some aspects of these developments. Similarly, see some recent works in this regard [30-33].

Now, we recall some basic definitions and properties of fractional calculus. Assume $\vartheta>0$. Then we define RL-fractional integral of the continuous function $z:[a, b] \rightarrow \mathbb{R}$ by

$$
\mathcal{I}_{0}^{\vartheta} z(t)=\frac{1}{\Gamma(\vartheta)} \int_{0}^{t}(t-\xi)^{\vartheta-1} z(\xi) \mathrm{d} \xi,
$$

such that the above integral exists $[34,35]$.

Let $r-1<\vartheta<r$. Then $r=[\vartheta]+1$. We define the Caputo derivative of the function $z \in C^{(r)}([a, b], \mathbb{R})$ as

$$
{ }^{c} \mathcal{D}_{0}^{\vartheta} z(t)=\frac{1}{\Gamma(r-\vartheta)} \int_{0}^{t}(t-\xi)^{r-\vartheta-1} z^{(r)}(\xi) \mathrm{d} \xi,
$$

such that the above integral exists [34,35]. It is known that for $n-1<\vartheta<n$, the solution of ${ }^{c} \mathcal{D}_{0}^{\vartheta} z(t)=0$ is $z(t)=m_{0}^{*}+m_{1}^{*} t+m_{2}^{*} t^{2}+\cdots+m_{n-1}^{*} t^{n-1}$; also, we have

$$
\mathcal{I}_{0}^{\vartheta}\left({ }^{c} \mathcal{D}_{0}^{\vartheta} z(t)\right)=z(t)+\sum_{j=0}^{n-1} m_{j}^{*} t^{j}=z(t)+m_{0}^{*}+m_{1}^{*} t+m_{2}^{*} t^{2}+\cdots+m_{n-1}^{*} t^{n-1},
$$

where $m_{0}^{*}, \ldots, m_{n-1}^{*} \in \mathbb{R}[36]$. The Krasnoselskii and Scheafer fixed point theorems are main tools which we use for our analysis.

Lemma 1. [37] We assume $\mathcal{E} \neq \varnothing$ as bounded, convex closed set in the Banach space $\mathcal{M}$. Consider $Y_{1}$ and $Y_{2}$ on $\mathcal{E}$ so that $Y_{1} z+Y_{2} w \in \mathcal{E}$ whenever $z, w \in \mathcal{E}$, and (a) the operator $Y_{1}$ is compact and continuous, (b) the operator $\mathrm{Y}_{2}$ is a contraction. Then $\exists y \in \mathcal{E}$ with $y=\mathrm{Y}_{1} y+\mathrm{Y}_{2} y$.

Lemma 2. [37] Consider $\mathcal{M}$ as a Banach space and a completely continuous mapping $\mathrm{Y}$ on $\mathcal{M}$. Then $\{z \in \mathcal{M}: z=v Y z, v \in(0,1)\}$ is not bounded or there exist a fixed point of $\mathrm{Y}$ inn $\mathcal{M}$.

\section{Main Theorems Regarding the Existence}

The present section is assigned to the results of existence theory on the graph of methylpropane (Figure 6). To begin it, we consider the Banach spaces $\mathcal{M}_{j}=\left\{z_{j}: z_{j}, z_{j}^{\prime} \in\right.$ $C[0,1]\}$ with $\left\|z_{j}\right\|_{\mathcal{M}_{j}}=\sup _{t \in[0,1]}\left|z_{j}(t)\right|+\sup _{t \in[0,1]}\left|z_{j}^{\prime}(t)\right|$ for $j \in \mathbb{N}_{1}^{13}$. Then the product space $\mathcal{M}=\left(\mathcal{M}_{1}, \mathcal{M}_{2}, \ldots, \mathcal{M}_{13}\right)$ equipped with the norm $\left\|z=\left(z_{1}, z_{2}, \ldots, z_{13}\right)\right\|_{\mathcal{M}}=$ $\sum_{j=1}^{13}\left\|z_{j}\right\|_{\mathcal{M}_{j}}$ is a Banach space.

Lemma 3. Let $\varphi_{j} \in C[0,1], j \in \mathbb{N}_{1}^{13}, \vartheta \in(1,2)$ and $\beta \in(0,1)$. Then $z_{j}$ is a solution of the $B V P s$

$$
\left\{\begin{array}{l}
{ }^{c} \mathcal{D}_{0}^{\vartheta} z_{j}(t)=\varphi_{j}(t), \\
z_{j}(0)+{ }^{c} \mathcal{D}_{0}^{\beta} z_{j}(0)+z_{j}^{\prime}(1)=0, \\
\int_{0}^{1}\left[z_{j}(\xi)+{ }^{c} \mathcal{D}_{0}^{\beta} z_{j}(\xi)+z_{j}^{\prime}(\xi)\right] \mathrm{d} \xi=0,
\end{array}\right.
$$


which is given by

$$
\begin{aligned}
z_{j}(t) & =\int_{0}^{t} \frac{(t-\xi)^{\vartheta-1}}{\Gamma(\vartheta)} \varphi_{j}(\xi) \mathrm{d} \xi+\frac{t-\Delta}{\Delta-1} \int_{0}^{1} \frac{(1-\xi)^{\vartheta-2}}{\Gamma(\vartheta-1)} \varphi_{j}(\xi) \mathrm{d} \xi \\
& +\frac{1-t}{\Delta-1}\left[\int_{0}^{1} \int_{0}^{\xi} \frac{(\xi-m)^{\vartheta-1}}{\Gamma(\vartheta)} \varphi_{j}(m) \mathrm{d} m \mathrm{~d} \xi+\int_{0}^{1} \int_{0}^{\xi} \frac{(\xi-m)^{\vartheta-2}}{\Gamma(\vartheta-1)} \varphi_{j}(m) \mathrm{d} m \mathrm{~d} \xi\right. \\
& \left.+\int_{0}^{1} \int_{0}^{\xi} \frac{(\xi-m)^{\vartheta-\beta-1}}{\Gamma(\vartheta-\beta)} \varphi_{j}(m) \mathrm{d} m \mathrm{~d} \xi\right]
\end{aligned}
$$

where $\Delta=\frac{3 \Gamma(3-\beta)+2}{2 \Gamma(3-\beta)} \neq 1$ and $j \in \mathbb{N}_{1}^{13}$.

Proof. Consider $z_{j}$ as the solution of the problems (4) $\left(j \in \mathbb{N}_{1}^{13}\right)$. So by (3), there are constants $a_{0}^{(j)}, a_{1}^{(j)} \in \mathbb{R}$ such that $z_{j}(t)=\mathcal{I}_{0}^{\vartheta} \varphi_{j}(t)+a_{0}^{(j)}+a_{1}^{(j)} t$, i.e.,

$$
z_{j}(t)=\int_{0}^{t} \frac{(t-\xi)^{\vartheta-1}}{\Gamma(\vartheta)} \varphi_{j}(\xi) \mathrm{d} \xi+a_{0}^{(j)}+a_{1}^{(j)} t
$$

Thus, we obtain $z_{j}^{\prime}(t)=\int_{0}^{t} \frac{(t-\xi)^{\vartheta-2}}{\Gamma(\vartheta-1)} \varphi_{j}(\xi) \mathrm{d} \xi+a_{1}^{(j)}$ and

$$
{ }^{c} \mathcal{D}_{0}^{\beta} z_{j}(t)=\int_{0}^{t} \frac{(t-\xi))^{\vartheta-\beta-1}}{\Gamma(\vartheta-\beta)} \varphi_{j}(\xi) \mathrm{d} \xi+a_{1}^{(j)} \frac{t^{1-\beta}}{\Gamma(2-\beta)},
$$

and so

$$
\begin{aligned}
& \int_{0}^{1} z_{j}(\xi) \mathrm{d} \xi=\int_{0}^{1} \int_{0}^{\xi} \frac{(\xi-m)^{\vartheta-1}}{\Gamma(\vartheta)} \varphi_{j}(m) \mathrm{d} m \mathrm{~d} \xi+a_{0}^{(j)}+\frac{1}{2} a_{1}^{(j)} \\
& \int_{0}^{1} z_{j}^{\prime}(\xi) \mathrm{d} \xi=\int_{0}^{1} \int_{0}^{\xi} \frac{(\xi-m)^{\vartheta-2}}{\Gamma(\vartheta-1)} \varphi_{j}(m) \mathrm{d} m \mathrm{~d} \xi+a_{1}^{(j)} \\
& \int_{0}^{1}{ }^{c} \mathcal{D}_{0}^{\beta} z_{j}(\xi) \mathrm{d} \xi=\int_{0}^{1} \int_{0}^{\xi} \frac{(\xi-m)^{\vartheta-\beta-1}}{\Gamma(\vartheta-\beta)} \varphi_{j}(m) \mathrm{d} m \mathrm{~d} \xi+a_{1}^{(j)} \frac{1}{\Gamma(3-\beta)} .
\end{aligned}
$$

By using the first boundary condition, we obtain

$$
a_{0}^{(j)}+a_{1}^{(j)}+\int_{0}^{1} \frac{(1-\xi)^{\vartheta-2}}{\Gamma(\vartheta-1)} \varphi_{j}(\xi) \mathrm{d} \xi=0 .
$$

Further, by the linearity of the integral in the second boundary condition, we have

$$
\int_{0}^{1} z_{j}(\xi) \mathrm{d} \xi+\int_{0}^{1}{ }^{c} \mathcal{D}_{0}^{\beta} z_{j}(\xi) \mathrm{d} \xi+\int_{0}^{1} z_{j}^{\prime}(\xi) \mathrm{d} \xi=0,
$$

and it gives

$$
\begin{gathered}
\int_{0}^{1} \int_{0}^{\xi} \frac{(\xi-m)^{\vartheta-1}}{\Gamma(\vartheta)} \varphi_{j}(m) \mathrm{d} m \mathrm{~d} \xi+a_{0}^{(j)}+\frac{1}{2} a_{1}^{(j)}+\int_{0}^{1} \int_{0}^{\xi} \frac{(\xi-m)^{\vartheta-\beta-1}}{\Gamma(\vartheta-\beta)} \varphi_{j}(m) \mathrm{d} m \mathrm{~d} \xi \\
+a_{1}^{(j)} \frac{1}{\Gamma(3-\beta)}+\int_{0}^{1} \int_{0}^{\xi} \frac{(\xi-m)^{\vartheta-2}}{\Gamma(\vartheta-1)} \varphi_{j}(m) \mathrm{d} m \mathrm{~d} \xi+a_{1}^{(j)}=0 .
\end{gathered}
$$


By some simple calculations on two latter Equations (7) and (8), and by considering the constant $\Delta=\frac{3 \Gamma(3-\beta)+2}{2 \Gamma(3-\beta)} \neq 1$, we obtain

$$
\begin{aligned}
a_{1}^{(j)} & =\frac{1}{(\Delta-1)}\left[\int_{0}^{1} \frac{(1-\xi)^{\vartheta-2}}{\Gamma(\vartheta-1)} \varphi_{j}(\xi) \mathrm{d} \xi-\int_{0}^{1} \int_{0}^{\xi} \frac{(\xi-m)^{\vartheta-1}}{\Gamma(\vartheta)} \varphi_{j}(m) \mathrm{d} m \mathrm{~d} \xi\right. \\
& \left.-\int_{0}^{1} \int_{0}^{\xi} \frac{(\xi-m)^{\vartheta-2}}{\Gamma(\vartheta-1)} \varphi_{j}(m) \mathrm{d} m \mathrm{~d} \xi-\int_{0}^{1} \int_{0}^{\xi} \frac{(\xi-m)^{\vartheta-\beta-1}}{\Gamma(\vartheta-\beta)} \varphi_{j}(m) \mathrm{d} m \mathrm{~d} \xi\right] .
\end{aligned}
$$

Then by substituting $a_{1}^{(j)}$ into (7), we obtain

$$
\begin{aligned}
a_{0}^{(j)} & =-\int_{0}^{1} \frac{(1-\xi)^{\vartheta-2}}{\Gamma(\vartheta-1)} \varphi_{j}(\xi) \mathrm{d} \xi \\
& -\frac{1}{(\Delta-1)}\left[\int_{0}^{1} \frac{(1-\xi)^{\vartheta-2}}{\Gamma(\vartheta-1)} \varphi_{j}(\xi) \mathrm{d} \xi-\int_{0}^{1} \int_{0}^{\xi} \frac{(\xi-m)^{\vartheta-1}}{\Gamma(\vartheta)} \varphi_{j}(m) \mathrm{d} m \mathrm{~d} \xi\right. \\
& \left.-\int_{0}^{1} \int_{0}^{\xi} \frac{(\xi-m)^{\vartheta-2}}{\Gamma(\vartheta-1)} \varphi_{j}(m) \mathrm{d} m \mathrm{~d} \xi-\int_{0}^{1} \int_{0}^{\xi} \frac{(\xi-m)^{\vartheta-\beta-1}}{\Gamma(\vartheta-\beta)} \varphi_{j}(m) \mathrm{d} m \mathrm{~d} \xi\right] .
\end{aligned}
$$

Next, we substitute the values of $a_{0}^{(j)}$ and $a_{1}^{(j)}$ into Equation (6). In this case, we see that $z_{j}$ satisfies (5). This completes the proof.

Here by considering (3), define the operator $\mathcal{T}$ on $\mathcal{M}$ by

$$
\mathcal{T}\left(z_{1}, z_{2}, \ldots, z_{13}\right)(t):=\left(\left(\mathcal{T}_{1} z_{1}\right)(t), \ldots,\left(\mathcal{T}_{13} z_{13}\right)(t)\right),
$$

where $\left(\mathcal{T}_{j}: \mathcal{M}_{j} \rightarrow \mathcal{M}_{j}\right)$,

$$
\begin{aligned}
\left(\mathcal{T}_{j} z_{j}\right)(t) & =\int_{0}^{t} \frac{(t-\xi)^{\vartheta-1}}{\Gamma(\vartheta)}\left[h_{j}\left(\xi, z_{j}(\xi), z_{j}^{\prime}(\xi)\right)+k_{j}\left(\xi, z_{j}(\xi), z_{j}^{\prime}(\xi)\right)\right] \mathrm{d} \xi \\
& +\frac{t-\Delta}{\Delta-1} \int_{0}^{1} \frac{(1-\xi)^{\vartheta-2}}{\Gamma(\vartheta-1)}\left[h_{j}\left(\xi, z_{j}(\xi), z_{j}^{\prime}(\xi)\right)+k_{j}\left(\xi, z_{j}(\xi), z_{j}^{\prime}(\xi)\right)\right] \mathrm{d} \xi \\
& +\frac{1-t}{\Delta-1}\left[\int_{0}^{1} \int_{0}^{\xi} \frac{(\xi-m)^{\vartheta-1}}{\Gamma(\vartheta)}\left[h_{j}\left(m, z_{j}(m), z_{j}^{\prime}(m)\right)+k_{j}\left(m, z_{j}(m), z_{j}^{\prime}(m)\right)\right] \mathrm{d} m \mathrm{~d} \xi\right. \\
& +\int_{0}^{1} \int_{0}^{\xi} \frac{(\xi-m)^{\vartheta-2}}{\Gamma(\vartheta-1)}\left[h_{j}\left(m, z_{j}(m), z_{j}^{\prime}(m)\right)+k_{j}\left(m, z_{j}(m), z_{j}^{\prime}(m)\right)\right] \mathrm{d} m \mathrm{~d} \xi \\
& \left.+\int_{0}^{1} \int_{0}^{\xi} \frac{(\xi-m)^{\vartheta-\beta-1}}{\Gamma(\vartheta-\beta)}\left[h_{j}\left(m, z_{j}(m), z_{j}^{\prime}(m)\right)+k_{j}\left(m, z_{j}(m), z_{j}^{\prime}(m)\right)\right] \mathrm{d} m \mathrm{~d} \xi\right],
\end{aligned}
$$

for all $t \in[0,1]$ and $z_{j} \in \mathcal{M}_{j}$. For convenience in writing, put

$$
\mathcal{K}_{0}^{*}:=\frac{1}{|\Delta-1| \Gamma(\vartheta+2)}+\frac{|\Delta-1|+1}{|\Delta-1| \Gamma(\vartheta+1)}+\frac{1+|\Delta|}{|\Delta-1| \Gamma(\vartheta)}+\frac{1}{|\Delta-1| \Gamma(\vartheta-\beta+2)},
$$




$$
\mathcal{K}_{1}^{*}:=\frac{|\Delta-1|+1}{|\Delta-1| \Gamma(\vartheta)}+\frac{1}{|\Delta-1| \Gamma(\vartheta+2)}+\frac{1}{|\Delta-1| \Gamma(\vartheta-\beta+2)}+\frac{1}{|\Delta-1| \Gamma(\vartheta+1)} .
$$

We now analyze the fractional BVP (2) with different conditions by terms of the existence criterion.

Theorem 1. Let $h_{1}, \ldots, h_{13}:[0,1] \times \mathbb{R}^{2} \rightarrow \mathbb{R}$ and $k_{1}, \ldots, k_{13}:[0,1] \times \mathbb{R}^{2} \rightarrow \mathbb{R}$ be continuous functions. Suppose that there exist continuous maps $\delta_{1}, \ldots, \delta_{13}:[0,1] \rightarrow \mathbb{R}^{+}$and $\mu_{1}, \ldots, \mu_{13}$ : $[0,1] \rightarrow \mathbb{R}^{+}$, and continuous non-decreasing maps, $\phi_{1}, \ldots, \phi_{13}: \mathbb{R}^{+} \rightarrow \mathbb{R}^{+}$and $\psi_{1}, \ldots, \chi_{13}$ : $\mathbb{R}^{+} \rightarrow \mathbb{R}^{+}$s.t.

$$
\left|h_{j}\left(t, z_{1}, z_{2}\right)\right| \leq \delta_{j}(t) \phi_{j}\left(\sum_{i=1}^{2}\left|z_{i}\right|\right), \quad\left|k_{j}\left(t, z_{1}, z_{2}\right)\right| \leq \mu_{j}(t) \chi_{j}\left(\sum_{i=1}^{2}\left|z_{i}\right|\right) .
$$

In addition, assume that continuous maps $\sigma_{1}, \ldots, \sigma_{13}:[0,1] \rightarrow \mathbb{R}$ exist s.t.

$$
\left|k_{j}\left(t, z_{1}, z_{2}\right)-k_{j}\left(t, q_{1}, q_{2}\right)\right| \leq \sigma_{j}(t)\left(\left|z_{1}-q_{1}\right|+\left|z_{2}-q_{2}\right|\right),
$$

for all $t \in[0,1], z_{1}, z_{2}, q_{1}, q_{2} \in \mathbb{R}$ and $j \in \mathbb{N}_{1}^{13}$. If $\lambda^{*}:=\left(\mathcal{K}_{0}^{*}+\mathcal{K}_{1}^{*}\right) \sum_{j=1}^{13}\left\|\sigma_{j}\right\|<1$, then the fractional BVPs (2) has a solution, where $\left\|\sigma_{j}\right\|=\sup _{t \in[0,1]}\left|\sigma_{j}(t)\right|$, and $\mathcal{K}_{0}^{*}$ and $\mathcal{K}_{1}^{*}$ are given in (10).

Proof. Set $\left\|\delta_{j}\right\|=\sup _{t \in[0,1]}\left|\delta_{j}(t)\right|$ and $\left\|\mu_{j}\right\|=\sup _{t \in[0,1]}\left|\mu_{j}(t)\right|$. We select the appropriate constant $\rho$ so that

$$
\rho \geq \sum_{j=1}^{13}\left(\phi_{j}\left(\left\|z_{j}\right\|_{\mathcal{M}_{j}}\right)\left\|\delta_{j}\right\|+\chi_{j}\left(\left\|z_{j}\right\|_{\mathcal{M}_{j}}\right)\left\|\mu_{j}\right\|\right)\left\{\mathcal{K}_{0}^{*}+\mathcal{K}_{1}^{*}\right\}
$$

where $\mathcal{K}_{i}^{* \prime}$ are given in (10). Define subsets

$$
\mathcal{B}_{\rho}:=\left\{z=\left(z_{1}, z_{2}, \ldots, z_{13}\right) \in \mathcal{M}=\left(\mathcal{M}_{1}, \mathcal{M}_{2}, \ldots, \mathcal{M}_{13}\right):\|z\|_{\mathcal{M}} \leq \rho\right\},
$$

where $\rho$ is given in (11). Define $\mathcal{T}_{1}$ and $\mathcal{T}_{2}$ on $\mathcal{B}_{\rho}$ as

$$
\begin{aligned}
& \mathcal{T}_{1}\left(z_{1}, z_{2}, \ldots, z_{13}\right)(t):=\left(\left(\mathcal{T}_{1}^{(1)} z_{1}\right)(t),\left(\mathcal{T}_{1}^{(2)} z_{2}\right)(t), \ldots,\left(\mathcal{T}_{1}^{(13)} z_{13}\right)(t)\right), \\
& \mathcal{T}_{2}\left(z_{1}, z_{2}, \ldots, z_{13}\right)(t):=\left(\left(\mathcal{T}_{2}^{(1)} z_{1}\right)(t),\left(\mathcal{T}_{2}^{(2)} z_{2}\right)(t), \ldots,\left(\mathcal{T}_{2}^{(13)} z_{13}\right)(t)\right),
\end{aligned}
$$

where

$$
\begin{aligned}
\left(\mathcal{T}_{1}^{(j)} z_{j}\right)(t) & =\int_{0}^{t} \frac{(t-\xi)^{\vartheta-1}}{\Gamma(\vartheta)} h_{j}\left(\xi, z_{j}(\xi), z_{j}^{\prime}(\xi)\right) \mathrm{d} \xi \\
& +\frac{t-\Delta}{\Delta-1} \int_{0}^{1} \frac{(1-\xi)^{\vartheta-2}}{\Gamma(\vartheta-1)} h_{j}\left(\xi, z_{j}(\xi), z_{j}^{\prime}(\xi)\right) \mathrm{d} \xi \\
& +\frac{1-t}{\Delta-1}\left[\int_{0}^{1} \int_{0}^{\xi} \frac{(\xi-m)^{\vartheta-1}}{\Gamma(\vartheta)} h_{j}\left(m, z_{j}(m), z_{j}^{\prime}(m)\right) \mathrm{d} m \mathrm{~d} \xi\right. \\
& +\int_{0}^{1} \int_{0}^{\xi} \frac{(\xi-m)^{\vartheta-2}}{\Gamma(\vartheta-1)} h_{j}\left(m, z_{j}(m), z_{j}^{\prime}(m)\right) \mathrm{d} m \mathrm{~d} \xi \\
& \left.+\int_{0}^{1} \int_{0}^{\xi} \frac{(\xi-m)^{\vartheta-\beta-1}}{\Gamma(\vartheta-\beta)} h_{j}\left(m, z_{j}(m), z_{j}^{\prime}(m)\right) \mathrm{d} m \mathrm{~d} \xi\right]
\end{aligned}
$$


and

$$
\begin{aligned}
\left(\mathcal{T}_{2}^{(j)} z_{j}\right)(t) & =\int_{0}^{t} \frac{(t-\xi)^{\vartheta-1}}{\Gamma(\vartheta)} k_{j}\left(\xi, z_{j}(\xi), z_{j}^{\prime}(\xi)\right) \mathrm{d} \xi \\
& +\frac{t-\Delta}{\Delta-1} \int_{0}^{1} \frac{(1-\xi)^{\vartheta-2}}{\Gamma(\vartheta-1)} k_{j}\left(\xi, z_{j}(\xi), z_{j}^{\prime}(\xi)\right) \mathrm{d} \xi \\
& +\frac{1-t}{\Delta-1}\left[\int_{0}^{1} \int_{0}^{\xi} \frac{(\xi-m)^{\vartheta-1}}{\Gamma(\vartheta)} k_{j}\left(m, z_{j}(m), z_{j}^{\prime}(m)\right) \mathrm{d} m \mathrm{~d} \xi\right. \\
& +\int_{0}^{1} \int_{0}^{\xi} \frac{(\xi-m)^{\vartheta-2}}{\Gamma(\vartheta-1)} k_{j}\left(m, z_{j}(m), z_{j}^{\prime}(m)\right) \mathrm{d} m \mathrm{~d} \xi \\
& \left.+\int_{0}^{1} \int_{0}^{\xi} \frac{(\xi-m)^{\vartheta-\beta-1}}{\Gamma(\vartheta-\beta)} k_{j}\left(m, z_{j}(m), z_{j}^{\prime}(m)\right) \mathrm{d} m \mathrm{~d} \xi\right]
\end{aligned}
$$

for each $t \in[0,1]$ and $z_{j} \in \mathcal{M}_{j}$. Assume that $\phi_{j}^{*}=\sup _{z_{j} \in \mathcal{M}_{j}} \phi_{j}\left(\left\|z_{j}\right\|_{\mathcal{M}_{j}}\right)$ and $\chi_{j}^{*}=$ $\sup _{z_{j} \in \mathcal{M}_{j}} \chi_{j}\left(\left\|z_{j}\right\|_{\mathcal{M}_{j}}\right)$. Now for every $z=\left(z_{1}, \ldots, z_{13}\right), q=\left(q_{1}, \ldots, q_{13}\right) \in \mathcal{B}_{\rho}$, we have

$$
\begin{aligned}
& \left|\left(\mathcal{T}_{1}^{(j)} z_{j}+\mathcal{T}_{2}^{(j)} q_{j}\right)(t)\right| \leq \int_{0}^{t} \frac{(t-\xi)^{\vartheta-1}}{\Gamma(\vartheta)}\left[\left|h_{j}\left(\xi, z_{j}(\xi), z_{j}^{\prime}(\xi)\right)\right|+\left|k_{j}\left(\xi, q_{j}(\xi), q_{j}^{\prime}(\xi)\right)\right|\right] \mathrm{d} \xi \\
& +\frac{t+|\Delta|}{|\Delta-1|} \int_{0}^{1} \frac{(1-\xi)^{\vartheta-2}}{\Gamma(\vartheta-1)}\left[\left|h_{j}\left(\xi, z_{j}(\xi), z_{j}^{\prime}(\xi)\right)\right|+\left|k_{j}\left(\xi, q_{j}(\xi), q_{j}^{\prime}(\xi)\right)\right|\right] \mathrm{d} \xi \\
& +\frac{1-t}{|\Delta-1|}\left(\int_{0}^{1} \int_{0}^{\xi} \frac{(\xi-m)^{\vartheta-1}}{\Gamma(\vartheta)}\left[\left|h_{j}\left(m, z_{j}(m), z_{j}^{\prime}(m)\right)\right|+\left|k_{j}\left(m, q_{j}(m), q_{j}^{\prime}(m)\right)\right|\right] \mathrm{d} m \mathrm{~d} \xi\right. \\
& +\int_{0}^{1} \int_{0}^{\xi} \frac{(\xi-m)^{\vartheta-2}}{\Gamma(\vartheta-1)}\left[\left|h_{j}\left(m, z_{j}(m), z_{j}^{\prime}(m)\right)\right|+\left|k_{j}\left(m, q_{j}(m), q_{j}^{\prime}(m)\right)\right|\right] \mathrm{d} m \mathrm{~d} \xi \\
& \left.+\int_{0}^{1} \int_{0}^{\xi} \frac{(\xi-m)^{\vartheta-\beta-1}}{\Gamma(\vartheta-\beta)}\left[\left|h_{j}\left(m, z_{j}(m), z_{j}^{\prime}(m)\right)\right|+\left|k_{j}\left(m, q_{j}(m), q_{j}^{\prime}(m)\right)\right|\right] \mathrm{d} m \mathrm{~d} \xi\right) \\
& \leq \int_{0}^{t} \frac{(t-\xi)^{\vartheta-1}}{\Gamma(\vartheta)}\left[\delta_{j}(\xi) \phi_{j}\left(\left\|z_{j}\right\|_{\mathcal{M}_{j}}\right)+\mu_{j}(\xi) \chi_{j}\left(\left\|q_{j}\right\|_{\mathcal{M}_{j}}\right)\right] \mathrm{d} \xi \\
& +\frac{t+|\Delta|}{|\Delta-1|} \int_{0}^{1} \frac{(1-\xi))^{\vartheta-2}}{\Gamma(\vartheta-1)}\left[\delta_{j}(\xi) \phi_{j}\left(\left\|z_{j}\right\|_{\mathcal{M}_{j}}\right)+\mu_{j}(\xi) \chi_{j}\left(\left\|q_{j}\right\|_{\mathcal{M}_{j}}\right)\right] \mathrm{d} \xi \\
& +\frac{1-t}{|\Delta-1|}\left(\int_{0}^{1} \int_{0}^{\xi} \frac{(\xi-m)^{\vartheta-1}}{\Gamma(\vartheta)}\left[\delta_{j}(m) \phi_{j}\left(\left\|z_{j}\right\|_{\mathcal{M}_{j}}\right)+\mu_{j}(m) \chi_{j}\left(\left\|q_{j}\right\|_{\mathcal{M}_{j}}\right)\right] \mathrm{d} m \mathrm{~d} \xi\right. \\
& +\int_{0}^{1} \int_{0}^{\xi} \frac{(\xi-m)^{\vartheta-2}}{\Gamma(\vartheta-1)}\left[\delta_{j}(m) \phi_{j}\left(\left\|z_{j}\right\|_{\mathcal{M}_{j}}\right)+\mu_{j}(m) \chi_{j}\left(\left\|q_{j}\right\|_{\mathcal{M}_{j}}\right)\right] \mathrm{d} m \mathrm{~d} \xi
\end{aligned}
$$




$$
\begin{aligned}
& \left.+\int_{0}^{1} \int_{0}^{\xi} \frac{(\xi-m)^{\vartheta-\beta-1}}{\Gamma(\vartheta-\beta)}\left[\delta_{j}(m) \phi_{j}\left(\left\|z_{j}\right\|_{\mathcal{M}_{j}}\right)+\mu_{j}(m) \chi_{j}\left(\left\|q_{j}\right\|_{\mathcal{M}_{j}}\right)\right] \mathrm{d} m \mathrm{~d} \xi\right) \\
& \leq\left(\left\|\delta_{j}\right\| \phi_{j}^{*}+\left\|\mu_{j}\right\| \chi_{j}^{*}\right)\left[\frac{1}{\Gamma(\vartheta+1)}+\frac{1+|\Delta|}{|\Delta-1| \Gamma(\vartheta)}+\frac{1}{|\Delta-1| \Gamma(\vartheta+2)}\right. \\
& \left.+\frac{1}{|\Delta-1| \Gamma(\vartheta+1)}+\frac{1}{|\Delta-1| \Gamma(\vartheta-\beta+2)}\right] \\
& =\left(\left\|\delta_{j}\right\| \phi_{j}^{*}+\left\|\mu_{j}\right\| \chi_{j}^{*}\right) \mathcal{K}_{0}^{*} \\
& \text { and } \\
& \mid\left(\left(\mathcal{T}_{1}^{(j)} z_{j}\right)^{\prime}(t)+\left(\mathcal{T}_{2}^{(j)} q_{j}\right)^{\prime}(t) \mid \leq \int_{0}^{t} \frac{(t-\xi)^{\vartheta-2}}{\Gamma(\vartheta-1)}\left[\left|h_{j}\left(\xi, z_{j}(\xi), z_{j}^{\prime}(\xi)\right)\right|+\left|k_{j}\left(\xi, q_{j}(\xi), q_{j}^{\prime}(\xi)\right)\right|\right] \mathrm{d} \xi\right. \\
& +\frac{1}{|\Delta-1|} \int_{0}^{1} \frac{(1-\xi)^{\vartheta-2}}{\Gamma(\vartheta-1)}\left[\left|h_{j}\left(\xi, z_{j}(\xi), z_{j}^{\prime}(\xi)\right)\right|+\left|k_{j}\left(\xi, q_{j}(\xi), q_{j}^{\prime}(\xi)\right)\right|\right] \mathrm{d} \xi \\
& +\frac{1}{|\Delta-1|}\left(\int_{0}^{1} \int_{0}^{\xi} \frac{(\xi-m)^{\vartheta-1}}{\Gamma(\vartheta)}\left[\left|h_{j}\left(m, z_{j}(m), z_{j}^{\prime}(m)\right)\right|+\left|k_{j}\left(m, q_{j}(m), q_{j}^{\prime}(m)\right)\right|\right] \mathrm{d} m \mathrm{~d} \xi\right. \\
& +\int_{0}^{1} \int_{0}^{\xi} \frac{(\xi-m)^{\vartheta-2}}{\Gamma(\vartheta-1)}\left[\left|h_{j}\left(m, z_{j}(m), z_{j}^{\prime}(m)\right)\right|+\left|k_{j}\left(m, q_{j}(m), q_{j}^{\prime}(m)\right)\right|\right] \mathrm{d} m \mathrm{~d} \xi \\
& \left.+\int_{0}^{1} \int_{0}^{\xi} \frac{(\xi-m)^{\vartheta-\beta-1}}{\Gamma(\vartheta-\beta)}\left[\left|h_{j}\left(m, z_{j}(m), z_{j}^{\prime}(m)\right)\right|+\left|k_{j}\left(m, q_{j}(m), q_{j}^{\prime}(m)\right)\right|\right] \mathrm{d} m \mathrm{~d} \xi\right) \\
& \leq\left(\left\|\delta_{j}\right\| \phi_{j}^{*}+\left\|\mu_{j}\right\| \chi_{j}^{*}\right)\left[\frac{1}{\Gamma(\vartheta)}+\frac{1}{|\Delta-1| \Gamma(\vartheta)}+\frac{1}{|\Delta-1| \Gamma(\vartheta+2)}\right. \\
& \left.+\frac{1}{|\Delta-1| \Gamma(\vartheta+1)}+\frac{1}{|\Delta-1| \Gamma(\vartheta-\beta+2)}\right] \\
& =\left(\left\|\delta_{j}\right\| \phi_{j}^{*}+\left\|\mu_{j}\right\| \chi_{j}^{*}\right) \mathcal{K}_{1}^{*}
\end{aligned}
$$

This yields

$$
\left\|\mathcal{T}_{1} z+\mathcal{T}_{2} q\right\|_{\mathcal{M}}=\sum_{j=1}^{13}\left\|\mathcal{T}_{1}^{(j)} z_{j}+\mathcal{T}_{2}^{(j)} q_{j}\right\|_{\mathcal{M}_{j}} \leq \sum_{j=1}^{13}\left(\left\|\delta_{j}\right\| \phi_{j}^{*}+\left\|\mu_{j}\right\| \chi_{j}^{*}\right)\left(\mathcal{K}_{0}^{*}+\mathcal{K}_{1}^{*}\right) \leq \rho,
$$

and so $\left\|\mathcal{T}_{1} z+\mathcal{T}_{2} q\right\|_{\mathcal{M}} \leq \rho$ and $\mathcal{T}_{1} z+\mathcal{T}_{2} q \in \mathcal{B}_{\rho}$. The continuity of $\mathcal{T}_{1}$ is proved from the continuity of the functions $h_{j}$. We now prove the uniform boundedness of the operator $\mathcal{T}_{1}$. We have

$$
\begin{aligned}
\left|\left(\mathcal{T}_{1}^{(j)} z_{j}\right)(t)\right| & \leq \int_{0}^{t} \frac{(t-\xi)^{\vartheta-1}}{\Gamma(\vartheta)}\left|h_{j}\left(\xi, z_{j}(\xi), z_{j}^{\prime}(\xi)\right)\right| \mathrm{d} \xi \\
& +\frac{t+|\Delta|}{|\Delta-1|} \int_{0}^{1} \frac{(1-\xi)^{\vartheta-2}}{\Gamma(\vartheta-1)}\left|h_{j}\left(\xi, z_{j}(\xi), z_{j}^{\prime}(\xi)\right)\right| \mathrm{d} \xi
\end{aligned}
$$




$$
\begin{aligned}
& +\frac{1-t}{|\Delta-1|}\left[\int_{0}^{1} \int_{0}^{\xi} \frac{(\xi-m)^{\vartheta-1}}{\Gamma(\vartheta)}\left|h_{j}\left(m, z_{j}(m), z_{j}^{\prime}(m)\right)\right| \mathrm{d} m \mathrm{~d} \xi\right. \\
& +\int_{0}^{1} \int_{0}^{\xi} \frac{(\xi-m)^{\vartheta-2}}{\Gamma(\vartheta-1)}\left|h_{j}\left(m, z_{j}(m), z_{j}^{\prime}(m)\right)\right| \mathrm{d} m \mathrm{~d} \xi \\
& \left.+\int_{0}^{1} \int_{0}^{\xi} \frac{(\xi-m)^{\vartheta-\beta-1}}{\Gamma(\vartheta-\beta)}\left|h_{j}\left(m, z_{j}(m), z_{j}^{\prime}(m)\right)\right| \mathrm{d} m \mathrm{~d} \xi\right] \\
& \leq\left\|\delta_{j}\right\| \phi_{j}\left(\left|z_{j}(t)\right|+\left|z_{j}^{\prime}(t)\right|\right)\left[\frac{1+|\Delta|}{|\Delta-1| \Gamma(\vartheta)}+\frac{1}{|\Delta-1| \Gamma(\vartheta+2)}\right. \\
& \left.+\frac{|\Delta-1|+1}{|\Delta-1| \Gamma(\vartheta+1)}+\frac{1}{|\Delta-1| \Gamma(\vartheta-\beta+2)}\right] \\
& =\left\|\delta_{j}\right\| \phi_{j}(\rho) \mathcal{K}_{0}^{*} .
\end{aligned}
$$

Further, note that

$$
\left|\left(\mathcal{T}_{1}^{(j)} z_{j}\right)^{\prime}(t)\right| \leq\left\|\delta_{j}\right\| \phi_{j}(\rho) \mathcal{K}_{1}^{*}
$$

for all $z$ in $\mathcal{B}_{\rho}$. Thus,

$$
\left\|\mathcal{T}_{1} z\right\|_{\mathcal{M}}=\sum_{j=1}^{13}\left\|\mathcal{T}_{1}^{(j)} z_{j}\right\|_{\mathcal{M}_{j}} \leq \sum_{j=1}^{13}\left\|\delta_{j}\right\| \phi_{j}(\rho)\left(\mathcal{K}_{0}^{*}+\mathcal{K}_{1}^{*}\right)
$$

So $\mathcal{T}_{1}$ is uniformly bounded on $\mathcal{B}_{\rho}$. Now, we are going to show the compactness of the operator $\mathcal{T}_{1}$ on $\mathcal{B}_{\rho}$. To do this, let $0 \leq t_{1}, t_{2} \leq 1$ with $t_{1}<t_{2}$; therefore, we conclude

$$
\begin{aligned}
& \left|\left(\mathcal{T}_{1}^{(j)} z_{j}\right)\left(t_{2}\right)-\left(\mathcal{T}_{1}^{(j)} z_{j}\right)\left(t_{1}\right)\right| \leq \int_{0}^{t_{1}} \frac{\left[\left(t_{2}-\xi\right)^{\vartheta-1}-\left(t_{1}-\xi\right)^{\vartheta-1}\right]}{\Gamma(\vartheta)}\left|h_{j}\left(\xi, z_{j}(\xi), z_{j}^{\prime}(\xi)\right)\right| \mathrm{d} \xi \\
& +\int_{t_{1}}^{t_{2}} \frac{\left(t_{2}-\xi\right)^{\vartheta-1}}{\Gamma(\vartheta)}\left|h_{j}\left(\xi, z_{j}(\xi), z_{j}^{\prime}(\xi)\right)\right| \mathrm{d} \xi \\
& +\frac{\left(t_{2}-t_{1}\right)}{|\Delta-1|} \int_{0}^{1} \frac{(1-\xi)^{\vartheta-2}}{\Gamma(\vartheta-1)}\left|h_{j}\left(\xi, z_{j}(\xi), z_{j}^{\prime}(\xi)\right)\right| \mathrm{d} \xi \\
& +\frac{\left(t_{2}-t_{1}\right)}{|\Delta-1|}\left[\int_{0}^{1} \int_{0}^{\xi} \frac{(\xi-m)^{\vartheta-1}}{\Gamma(\vartheta)}\left|h_{j}\left(m, z_{j}(m), z_{j}^{\prime}(m)\right)\right| \mathrm{d} m \mathrm{~d} \xi\right. \\
& +\int_{0}^{1} \int_{0}^{\xi} \frac{(\xi-m)^{\vartheta-2}}{\Gamma(\vartheta-1)}\left|h_{j}\left(m, z_{j}(m), z_{j}^{\prime}(m)\right)\right| \mathrm{d} m \mathrm{~d} \xi \\
& \left.+\int_{0}^{1} \int_{0}^{\xi} \frac{(\xi-m)^{\vartheta-\beta-1}}{\Gamma(\vartheta-\beta)}\left|h_{j}\left(m, z_{j}(m), z_{j}^{\prime}(m)\right)\right| \mathrm{d} m \mathrm{~d} \xi\right] \\
& \leq \frac{\left\|\delta_{j}\right\| \phi_{j}(\rho)}{\Gamma(\vartheta+1)}\left[-\left(t_{2}-t_{1}\right)^{\vartheta}+\left(t_{2}-t_{1}\right)^{\vartheta}+t_{2}^{\vartheta}-t_{1}^{\vartheta}\right]+\frac{\left\|\delta_{j}\right\| \phi_{j}(\rho)\left(t_{2}-t_{1}\right)}{|\Delta-1| \Gamma(\vartheta)} \\
& +\frac{\left\|\delta_{j}\right\| \phi_{j}(\rho)\left(t_{2}-t_{1}\right)}{|\Delta-1|}\left[\frac{1}{\Gamma(\vartheta+2)}+\frac{1}{\Gamma(\vartheta+1)}+\frac{1}{\Gamma(\vartheta-\beta+2)}\right] .
\end{aligned}
$$


Hence, $\left|\left(\mathcal{T}_{1}^{(j)} z_{j}\right)\left(t_{2}\right)-\left(\mathcal{T}_{1}^{(j)} z_{j}\right)\left(t_{1}\right)\right| \rightarrow 0$ as $t_{1} \rightarrow t_{2}$. Similarly, one can see that

$$
\left|\left(\mathcal{T}_{1}^{(j)} z_{j}\right)^{\prime}\left(t_{2}\right)-\left(\mathcal{T}_{1}^{(j)} z_{j}\right)^{\prime}\left(t_{1}\right)\right| \rightarrow 0,
$$

as $t_{1} \rightarrow t_{2}$; therefore, we obtain $\left\|\left(\mathcal{T}_{1} z_{j}\right)\left(t_{2}\right)-\left(\mathcal{T}_{1} z_{j}\right)\left(t_{1}\right)\right\|_{\mathcal{X}}$ tends to zero as $t_{1} \rightarrow t_{2}$. Thus, $\mathcal{T}_{1}$ will be equi-continuous, and is compact on $\mathcal{B}_{\rho}$ due to the Arzela-Ascoli property. Further, take $z, q \in \mathcal{B}_{\rho}$. Therefore

$$
\begin{aligned}
\mid\left(\mathcal{T}_{2}^{(j)} z_{j}\right)(t) & -\left(\mathcal{T}_{2}^{(j)} q_{j}\right)(t)\left|\leq \int_{0}^{t} \frac{(t-\xi)^{\vartheta-1}}{\Gamma(\vartheta)}\right| k_{j}\left(\xi, z_{j}(\xi), z_{j}^{\prime}(\xi)\right)-k_{j}\left(\xi, q_{j}(\xi), q_{j}^{\prime}(\xi)\right) \mid \mathrm{d} \xi \\
& +\frac{t+|\Delta|}{|\Delta-1|} \int_{0}^{1} \frac{(1-\xi)^{\vartheta-2}}{\Gamma(\vartheta-1)}\left|k_{j}\left(\xi, z_{j}(\xi), z_{j}^{\prime}(\xi)\right)-k_{j}\left(\xi, q_{j}(\xi), q_{j}^{\prime}(\xi)\right)\right| \mathrm{d} \xi \\
& +\frac{1-t}{|\Delta-1|}\left(\int_{0}^{1} \int_{0}^{\xi} \frac{(\xi-m)^{\vartheta-1}}{\Gamma(\vartheta)}\left|k_{j}\left(m, z_{j}(m), z_{j}^{\prime}(m)\right)-k_{j}\left(m, q_{j}(m), q_{j}^{\prime}(m)\right)\right| \mathrm{d} m \mathrm{~d} \xi\right. \\
& +\int_{0}^{1} \int_{0}^{\xi} \frac{(\xi-m)^{\vartheta-2}}{\Gamma(\vartheta-1)}\left|k_{j}\left(m, z_{j}(m), z_{j}^{\prime}(m)\right)-k_{j}\left(m, q_{j}(m), q_{j}^{\prime}(m)\right)\right| \mathrm{d} m \mathrm{~d} \xi \\
& \left.+\int_{0}^{1} \int_{0}^{\xi} \frac{(\xi-m)^{\vartheta-\beta-1}}{\Gamma(\vartheta-\beta)}\left|k_{j}\left(m, z_{j}(m), z_{j}^{\prime}(m)\right)-k_{j}\left(m, q_{j}(m), q_{j}^{\prime}(m)\right)\right| \mathrm{d} m \mathrm{~d} \xi\right) \\
& \leq \int_{0}^{t} \frac{(t-\xi)^{\vartheta-1}}{\Gamma(\vartheta)} \sigma_{j}(\xi)\left(\left|z_{j}(\xi)-q_{j}(\xi)\right|+\left|z_{j}^{\prime}(\xi)-q_{j}^{\prime}(\xi)\right|\right) \mathrm{d} \xi \\
& +\frac{t+|\Delta|}{|\Delta-1|} \int_{0}^{1} \frac{(1-\xi)^{\vartheta-2}}{\Gamma(\vartheta-1)} \sigma_{j}(\xi)\left(\left|z_{j}(\xi)-q_{j}(\xi)\right|+\left|z_{j}^{\prime}(\xi)-q_{j}^{\prime}(\xi)\right|\right) \mathrm{d} \xi \\
& \left.+\int_{0}^{1} \int_{0}^{\xi} \frac{(\xi-m)^{\vartheta-\beta-1}}{\Gamma(\vartheta-\beta)} \sigma_{j}(m)\left(\left|z_{j}(m)-q_{j}(m)\right|+\left|z_{j}^{\prime}(m)-q_{j}^{\prime}(m)\right|\right) \mathrm{d} m \mathrm{~d} \xi\right) \\
& +\frac{1-t}{|\Delta-1|}\left(\int_{0}^{1} \int_{0}^{\xi} \frac{(\xi-m)^{\vartheta-1}}{\Gamma(\vartheta)} \sigma_{j}(m)\left(\left|z_{j}(m)-q_{j}(m)\right|+\left|z_{j}^{\prime}(m)-q_{j}^{\prime}(m)\right|\right) \mathrm{d} m \mathrm{~d} \xi\right. \\
& +\int_{0}^{1} \int_{0}^{\xi} \frac{(\xi-m)^{\vartheta-2}}{\Gamma(\vartheta-1)} \sigma_{j}(m)\left(\left|z_{j}(m)-q_{j}(m)\right|+\left|z_{j}^{\prime}(m)-q_{j}^{\prime}(m)\right|\right) \mathrm{d} m \mathrm{~d} \xi \\
& \operatorname{and} \text { in the similar manner, } \\
& \\
&
\end{aligned}
$$$$
\left|\left(\mathcal{T}_{2}^{(j)} z_{j}\right)^{\prime}(t)-\left(\mathcal{T}_{2}^{(j)} q_{j}\right)^{\prime}(t)\right| \leq\left\|\sigma_{j}\right\| \mathcal{K}_{1}^{*}\left\|z_{j}-q_{j}\right\|_{\mathcal{M}_{j}} .
$$

Therefore, we have that

$$
\left\|\mathcal{T}_{2} z-\mathcal{T}_{2} q\right\|_{\mathcal{M}}=\sum_{j=1}^{13}\left\|\mathcal{T}_{2}^{(j)} z_{j}-\mathcal{T}_{2}^{(j)} q_{j}\right\|_{\mathcal{M}_{j}} \leq\left(\mathcal{K}_{0}^{*}+\mathcal{K}_{1}^{*}\right) \sum_{j=1}^{13}\left\|\sigma_{j}\right\|\left\|z_{j}-q_{j}\right\|_{\mathcal{M}_{j^{\prime}}}
$$


and so

$$
\left\|\mathcal{T}_{2} z-\mathcal{T}_{2} q\right\|_{\mathcal{M}} \leq \lambda^{*}\|z-q\|_{\mathcal{M}} .
$$

Since $\lambda^{*}<1, \mathcal{T}_{2}$ is a contraction on $\mathcal{B}_{\rho}$. Utilizing Lemma 1 , we deduced that a fixed point of $\mathcal{T}$ exists that satisfies the fractional BVPs (2).

In order to demonstrate the validity of the aforementioned conclusion, we give an abstract example.

Example 1. Consider the multi-term multi-dimensional DEs

$$
\left\{\begin{array}{l}
{ }^{c} \mathcal{D}_{0}^{1.38} z_{1}(t)=h_{1}\left(t, z_{1}(t), z_{1}^{\prime}(t)\right)+k_{1}\left(t, z_{1}(t), z_{1}^{\prime}(t)\right), \\
{ }^{c} \mathcal{D}_{0}^{1.38} z_{2}(t)=h_{2}\left(t, z_{2}(t), z_{2}^{\prime}(t)\right)+k_{2}\left(t, z_{2}(t), z_{2}^{\prime}(t)\right), \\
{ }^{c} \mathcal{D}_{0}^{1.38} z_{3}(t)=h_{3}\left(t, z_{3}(t), z_{3}^{\prime}(t)\right)+k_{3}\left(t, z_{3}(t), z_{3}^{\prime}(t)\right), \\
{ }^{c} \mathcal{D}_{0}^{1.38} z_{4}(t)=h_{4}\left(t, z_{4}(t), z_{4}^{\prime}(t)\right)+k_{4}\left(t, z_{4}(t), z_{4}^{\prime}(t)\right),
\end{array}\right.
$$

under the integral $B C s$

$$
\left\{\begin{array}{l}
z_{j}(0)+{ }^{c} \mathcal{D}_{0}^{0.47} z_{j}(0)+z_{j}^{\prime}(1)=0, \quad(j=1,2,3,4) \\
\int_{0}^{1}\left[z_{j}(\xi)+{ }^{c} \mathcal{D}_{0}^{0.47} z_{j}(\xi)+z_{j}^{\prime}(\xi)\right] \mathrm{d} \xi=0
\end{array}\right.
$$

where $\vartheta=1.38, \beta=0.47, n=4$, and ${ }^{c} \mathcal{D}_{0}^{1.38}$ and ${ }^{c} \mathcal{D}_{0}^{0.47}$ are derivatives of order $\gamma \in\{1.38,0.47\}$ of the Caputo type. Set $h_{j} \in C\left([0,1] \times \mathbb{R}^{3}, \mathbb{R}\right),(j=1, \ldots, 4)$ by

$$
\left\{\begin{array}{l}
h_{1}(t, z(t), \ell(t))=\frac{|\sin z(t)|}{2+t}+\frac{|\ell(t)|}{t+2}, \\
h_{2}(t, z(t), \ell(t))=\frac{|z(t)|}{5+5|z(t)|}+\frac{0.4|\sin \ell(t)|}{2+2|\sin \ell(t)|}, \\
h_{3}(t, z(t), \ell(t))=\frac{(t+1)}{700}|\arctan z(t)|+\frac{(t+1)|\ell(t)|}{700+700|\ell(t)|}, \\
h_{4}(t, z(t), \ell(t))=\frac{e^{t}}{8}|z(t)|+0.125|\ell(t)| e^{t},
\end{array}\right.
$$

and also $k_{j} \in C\left([0,1] \times \mathbb{R}^{3}, \mathbb{R}\right),(j=1, \ldots, 4)$ by

$$
\left\{\begin{array}{l}
k_{1}(t, z(t), \ell(t))=\frac{t}{1000}\left(\frac{|z(t)|}{|z(t)|+1}+|\sin \ell(t)|\right), \\
k_{2}(t, z(t), \ell(t))=\frac{|\sin z(t)|}{200+t}+\frac{|\arctan \ell(t)|}{200+t}, \\
k_{3}(t, z(t), \ell(t))=0.01 t|z(t)|+\frac{t}{100}|\arctan \ell(t)|, \\
k_{4}(t, z(t), \ell(t))=\frac{4 t}{100} \frac{|z(t)|}{|z(t)|+1}+0.04 t \frac{|\ell(t)|}{1+|\ell(t)|} .
\end{array}\right.
$$

Define $\phi_{j}: \mathbb{R}^{+} \rightarrow \mathbb{R}$ as $\phi_{m}(z)=z$ for all $z \in \mathbb{R}^{+}$and $j=1, \ldots, 4$. Further, we know that for each nonnegative real number $z, \sin z \leq z$ and $\arctan z \leq z$; therefore, we have

$$
\left|h_{1}\left(t, z(t), z^{\prime}(t)\right)\right| \leq \frac{1}{2+t}\left(|\sin z|+\left|z^{\prime}\right|\right)
$$




$$
\begin{aligned}
& \leq \frac{1}{2+t}\left(|z|+\left|z^{\prime}\right|\right)=\frac{1}{2+t} \phi_{1}\left(|z|+\left|z^{\prime}\right|\right), \\
\left|h_{2}\left(t, z(t), z^{\prime}(t)\right)\right| & \leq \frac{1}{5}\left(|z|+\left|\sin z^{\prime}\right|\right) \\
& \leq \frac{1}{5}\left(|z|+\left|z^{\prime}\right|\right)=\frac{1}{5} \phi_{2}\left(|z|+\left|z^{\prime}\right|\right), \\
\left|h_{3}\left(t, z(t), z^{\prime}(t)\right)\right| & \leq \frac{t+1}{700}\left(|\arctan z|+\left|z^{\prime}\right|\right) \\
& \leq \frac{t+1}{700}\left(|z|+\left|z^{\prime}\right|\right)=\frac{t+1}{700} \phi_{3}\left(|z|+\left|z^{\prime}\right|\right), \\
\left|h_{4}\left(t, z(t), z^{\prime}(t)\right)\right| & \leq \frac{e^{t}}{8}\left(|z|+\left|z^{\prime}\right|\right) \\
& \leq \frac{e^{t}}{8}\left(|z|+\left|z^{\prime}\right|\right)=\frac{e^{t}}{8} \phi_{4}\left(|z|+\left|z^{\prime}\right|\right),
\end{aligned}
$$

in which for $j=1, \ldots, 4, \delta_{j} \in C\left([0,1], \mathbb{R}^{+}\right)$are given as $\delta_{1}(t)=\frac{1}{2+t}, \delta_{2}(t)=\frac{1}{5}, \delta_{3}(t)=\frac{t+1}{700}$, and $\delta_{4}(t)=\frac{1}{8} e^{t}$. Further, we define nondecreasing and continuous mappings $\chi_{j}: \mathbb{R}^{+} \rightarrow \mathbb{R}^{+}$by $\chi_{j}(z)=z$ for all $z \in \mathbb{R}^{+}$and $j=1, \ldots, 4$. Then we obtain

$$
\begin{aligned}
\left|k_{1}\left(t, z(t), z^{\prime}(t)\right)\right| & \leq \frac{t}{1000}\left(|z|+\left|\sin z^{\prime}\right|\right) \\
& \leq \frac{t}{1000}\left(|z|+\left|z^{\prime}\right|\right)=\frac{t}{1000} \chi_{1}\left(|z|+\left|z^{\prime}\right|\right), \\
\left|k_{2}\left(t, z(t), z^{\prime}(t)\right)\right| & \leq \frac{1}{200+t}\left(|\sin z|+\left|\arctan z^{\prime}\right|\right) \\
& \leq \frac{1}{200+t}\left(|z|+\left|z^{\prime}\right|\right)=\frac{1}{200+t} \chi_{2}\left(|z|+\left|z^{\prime}\right|\right), \\
\left|k_{3}\left(t, z(t), z^{\prime}(t)\right)\right| & \leq \frac{t}{100}\left(|z|+\left|\arctan z^{\prime}\right|\right) \\
& \leq \frac{t}{100}\left(|z|+\left|z^{\prime}\right|\right)=\frac{t}{100} \chi_{3}\left(|z|+\left|z^{\prime}\right|\right), \\
\left|k_{4}\left(t, z(t), z^{\prime}(t)\right)\right| & \leq \frac{4 t}{100}\left(|z|+\left|z^{\prime}\right|\right) \\
& \leq \frac{4 t}{100}\left(|z|+\left|z^{\prime}\right|\right)=\frac{4 t}{100} \chi_{4}\left(|z|+\left|z^{\prime}\right|\right),
\end{aligned}
$$

in which for $j=1, \ldots, 4, \mu_{j} \in C\left([0,1], \mathbb{R}^{+}\right)$are given as $\mu_{1}(t)=\frac{t}{1000}, \mu_{2}(t)=\frac{1}{200+t^{\prime}}$ $\mu_{3}(t)=\frac{t}{100}$, and $\mu_{4}(t)=\frac{4 t}{100}$. On the other hand, by the mean value theorem, we know that $|\sin a-\sin b| \leq|a-b|$ and $|\arctan a-\arctan b| \leq|a-b|$ for all $a, b \in \mathbb{R}$. Thus, for $z_{1}, z_{2}, \ell_{1}, \ell_{2} \in \mathbb{R}$, we estimate

$$
\begin{aligned}
\mid k_{1}\left(t, z_{1}(t), z_{2}(t)\right) & -k_{1}\left(t, \ell_{1}(t), \ell_{2}(t)\right) \mid \\
& \leq \frac{t}{1000}\left(\left|z_{1}(t)-\ell_{1}(t)\right|+\left|\sin z_{2}(t)-\sin \ell_{2}(t)\right|\right)
\end{aligned}
$$




$$
\begin{gathered}
\leq \frac{t}{1000}\left(\left|z_{1}(t)-\ell_{1}(t)\right|+\left|z_{2}(t)-\ell_{2}(t)\right|\right) \\
\left|k_{2}\left(t, z_{1}(t), z_{2}(t)\right)-k_{2}\left(t, \ell_{1}(t), \ell_{2}(t)\right)\right| \\
\leq \frac{1}{200+t}\left(\left|\sin z_{1}(t)-\sin \ell_{1}(t)\right|+\left|\arctan z_{2}(t)-\arctan \ell_{2}(t)\right|\right) \\
\leq \frac{1}{200+t}\left(\left|z_{1}(t)-\ell_{1}(t)\right|+\left|z_{2}(t)-\ell_{2}(t)\right|\right), \\
\left|k_{3}\left(t, z_{1}(t), z_{2}(t)\right)-k_{3}\left(t, \ell_{1}(t), \ell_{2}(t)\right)\right| \\
\leq \frac{t}{100}\left(\left|z_{1}(t)-\ell_{1}(t)\right|+\left|\arctan z_{2}(t)-\arctan \ell_{2}(t)\right|\right) \\
\leq \frac{t}{100}\left(\left|z_{1}(t)-\ell_{1}(t)\right|+\left|z_{2}(t)-\ell_{2}(t)\right|\right),
\end{gathered}
$$

and

$$
\begin{aligned}
\mid k_{4}\left(t, z_{1}(t), z_{2}(t)\right) & -k_{4}\left(t, \ell_{1}(t), \ell_{2}(t)\right) \mid \\
& \leq \frac{4 t}{100}\left(\left|z_{1}(t)-\ell_{1}(t)\right|+\left|z_{2}(t)-\ell_{2}(t)\right|\right) \\
& \leq \frac{4 t}{100}\left(\left|z_{1}(t)-\ell_{1}(t)\right|+\left|z_{2}(t)-\ell_{2}(t)\right|\right) .
\end{aligned}
$$

Hence, $\sigma_{1}(t)=\frac{t}{1000}, \sigma_{2}(t)=\frac{1}{200+t^{\prime}}, \sigma_{3}(t)=\frac{t}{100}$, and $\sigma_{4}(t)=\frac{4 t}{100}$, where $\left\|\sigma_{1}\right\|=$ $\frac{1}{1000},\left\|\sigma_{2}\right\|=\frac{1}{200},\left\|\sigma_{3}\right\|=\frac{1}{100},\left\|\sigma_{4}\right\|=\frac{4}{100}$, and so $\sum_{j=1}^{4}\left\|\sigma_{j}\right\| \simeq 0.056$. According to the obtained values, we obtain $\Delta \simeq 2.2364, \mathcal{K}_{0}^{*} \simeq 5.1371, \mathcal{K}_{1}^{*} \simeq 3.4112$, and so $\mathcal{K}_{0}^{*}+\mathcal{K}_{1}^{*} \simeq 8.5483$. Hence,

$$
\lambda^{*}:=\left(\mathcal{K}_{0}^{*}+\mathcal{K}_{1}^{*}\right) \sum_{j=1}^{4}\left\|\sigma_{j}\right\|=\left(\mathcal{K}_{0}^{*}+\mathcal{K}_{1}^{*}\right)\left(\left\|\sigma_{1}\right\|+\left\|\sigma_{2}\right\|+\left\|\sigma_{3}\right\|+\left\|\sigma_{4}\right\|\right) \simeq 0.4787048<1 .
$$
solution.

Application of Theorem 1 leads us to infer that the fractional BVPs (12) and (13) possesses a

In the following, we investigate a special case of the fractional BVPs (2) on the methylpropane graph (Figure 6). Assume that $k_{j}\left(t, z_{j}(t), z_{j}^{\prime}(t)\right)=0$ are constant functions. Then the fractional problems (2) are converted into the following BVPs on the methylpropane graph under the integral BCs

$$
\left\{\begin{array}{l}
{ }^{c} \mathcal{D}_{0}^{\vartheta} z_{j}(t)=h_{j}\left(t, z_{j}(t), z_{j}^{\prime}(t)\right), \quad\left(j \in \mathbb{N}_{1}^{13}\right) \\
z_{j}(0)+{ }^{c} \mathcal{D}_{0}^{\beta} z_{j}(0)+z_{j}^{\prime}(1)=0, \\
\int_{0}^{1}\left[z_{j}(\xi)+{ }^{c} \mathcal{D}_{0}^{\beta} z_{j}(\xi)+z_{j}^{\prime}(\xi)\right] \mathrm{d} \xi=0,
\end{array}\right.
$$


where $\vartheta \in(1,2)$, and $h_{j}:[0,1] \times \mathbb{R}^{2} \rightarrow \mathbb{R}$ are continuous functions. By utilizing the Scheafer's theorem, we prove our next existence criterion. Before that, with adequate regard for Lemma 3, define $\mathrm{Y}$ on $\mathcal{M}$ as

$$
\mathrm{Y}\left(z_{1}, z_{2}, \ldots, z_{13}\right)(t):=\left(\left(\mathrm{Y}_{1} z_{1}\right)(t), \ldots,\left(\mathrm{Y}_{13} z_{13}\right)(t)\right),
$$

where

$$
\begin{aligned}
\left(\mathrm{Y}_{j} z_{j}\right)(t) & =\int_{0}^{t} \frac{(t-\xi)^{\vartheta-1}}{\Gamma(\vartheta)} h_{j}\left(\xi, z_{j}(\xi), z_{j}^{\prime}(\xi)\right) \mathrm{d} \xi \\
& +\frac{t-\Delta}{\Delta-1} \int_{0}^{1} \frac{(1-\xi)^{\vartheta-2}}{\Gamma(\vartheta-1)} h_{j}\left(\xi, z_{j}(\xi), z_{j}^{\prime}(\xi)\right) \mathrm{d} \xi \\
& +\frac{1-t}{\Delta-1}\left[\int_{0}^{1} \int_{0}^{\xi} \frac{(\xi-m)^{\vartheta-1}}{\Gamma(\vartheta)} h_{j}\left(m, z_{j}(m), z_{j}^{\prime}(m)\right) \mathrm{d} m \mathrm{~d} \xi\right. \\
& +\int_{0}^{1} \int_{0}^{\xi} \frac{(\xi-m)^{\vartheta-2}}{\Gamma(\vartheta-1)} h_{j}\left(m, z_{j}(m), z_{j}^{\prime}(m)\right) \mathrm{d} m \mathrm{~d} \xi \\
& \left.+\int_{0}^{1} \int_{0}^{\xi} \frac{(\xi-m)^{\vartheta-\beta-1}}{\Gamma(\vartheta-\beta)} h_{j}\left(m, z_{j}(m), z_{j}^{\prime}(m)\right) \mathrm{d} m \mathrm{~d} \xi\right],
\end{aligned}
$$

for all $t \in[0,1], z_{j} \in \mathcal{M}_{j}$, and $j \in \mathbb{N}_{1}^{13}$. In the following, the existence property for FBVPs (14) are proved under the boundedness assumption for the continuous functions $h_{j}$.

Theorem 2. Regard the continuous functions $h_{1}, \ldots, h_{13}:[0,1] \times \mathbb{R}^{2} \rightarrow \mathbb{R}$. Assume that the constants $\varrho_{j}>0$ exist with $\left|h_{j}\left(t, z_{1}, z_{2}\right)\right| \leq \varrho_{j}$ for all $z_{1}, z_{2} \in \mathbb{R},\left(j \in \mathbb{N}_{1}^{13}\right)$ and $\forall t \in[0,1]$. Then the FBVPs (14) have solutions on each edge of the methylpropane graph.

Proof. Regarding the definition of operator Y, clearly, the fractional BVPs (14) have solutions if and only if there exist a fixed point of $Y$ on $\mathcal{M}=\mathcal{M}_{1} \times \cdots \times \mathcal{M}_{13}$. Initially, we check that $\mathrm{Y}$ is complete continuous. Continuity of $h_{1}, \ldots, h_{13}$ implies that of $\mathrm{Y}: \mathcal{M} \rightarrow \mathcal{M}$. Assume a bounded set $\mathcal{O}$ in $\mathcal{M}$ and $z_{j} \in \mathcal{M}_{j}$. Then we have

$$
\begin{aligned}
\left|\left(Y_{j} z_{j}\right)(t)\right| & \leq \int_{0}^{t} \frac{(t-\xi)^{\vartheta-1}}{\Gamma(\vartheta)}\left|h_{j}\left(\xi, z_{j}(\xi), z_{j}^{\prime}(\xi)\right)\right| \mathrm{d} \xi \\
& +\frac{t+|\Delta|}{|\Delta-1|} \int_{0}^{1} \frac{(1-\xi)^{\vartheta-2}}{\Gamma(\vartheta-1)}\left|h_{j}\left(\xi, z_{j}(\xi), z_{j}^{\prime}(\xi)\right)\right| \mathrm{d} \xi \\
& +\frac{1-t}{|\Delta-1|}\left[\int_{0}^{1} \int_{0}^{\xi} \frac{(\xi-m)^{\vartheta-1}}{\Gamma(\vartheta)}\left|h_{j}\left(m, z_{j}(m), z_{j}^{\prime}(m)\right)\right| \mathrm{d} m \mathrm{~d} \xi\right. \\
& +\int_{0}^{1} \int_{0}^{\xi} \frac{(\xi-m)^{\vartheta-2}}{\Gamma(\vartheta-1)}\left|h_{j}\left(m, z_{j}(m), z_{j}^{\prime}(m)\right)\right| \mathrm{d} m \mathrm{~d} \xi \\
& \left.+\int_{0}^{1} \int_{0}^{\xi} \frac{(\xi-m)^{\vartheta-\beta-1}}{\Gamma(\vartheta-\beta)}\left|h_{j}\left(m, z_{j}(m), z_{j}^{\prime}(m)\right)\right| \mathrm{d} m \mathrm{~d} \xi\right]
\end{aligned}
$$




$$
\begin{aligned}
& \leq \varrho_{j}\left[\frac{1+|\Delta|}{|\Delta-1| \Gamma(\vartheta)}+\frac{1}{|\Delta-1| \Gamma(\vartheta+2)}+\frac{|\Delta-1|+1}{|\Delta-1| \Gamma(\vartheta+1)}+\frac{1}{|\Delta-1| \Gamma(\vartheta-\beta+2)}\right] \\
& =\varrho_{j} \mathcal{K}_{0}^{*},
\end{aligned}
$$

$\forall t \in[0,1]$, and $\mathcal{K}_{0}^{*}$ is introduced in (10). Similarly,

$$
\begin{aligned}
& \left|\left(\mathrm{Y}_{j} z_{j}\right)^{\prime}(t)\right| \leq \varrho_{j}\left[\frac{|\Delta-1|+1}{|\Delta-1| \Gamma(\vartheta)}+\frac{1}{|\Delta-1| \Gamma(\vartheta+1)}+\frac{1}{|\Delta-1| \Gamma(\vartheta-\beta+2)}+\frac{1}{|\Delta-1| \Gamma(\vartheta+2)}\right]=\varrho_{j} \mathcal{K}_{1}^{*}, \\
& \forall t \in[0,1] \text {, in which } \mathcal{K}_{1}^{*} \text { is as (10). Thus }\left\|\mathrm{Y}_{j} z_{j}(t)\right\|_{\mathcal{M}_{j}} \leq \varrho_{j}\left(\mathcal{K}_{0}^{*}+\mathcal{K}_{1}^{*}\right) \text {, and }\|\mathrm{Y} z(t)\|_{\mathcal{M}}= \\
& \left|\left(\mathrm{Y}_{j} z_{j}\right)\left(t_{2}\right)-\left(\mathrm{Y}_{j} z_{j}\right)\left(t_{1}\right)\right| \leq \int_{0}^{t_{1}} \frac{\left[\left(t_{2}-\xi\right)^{\vartheta-1}-\left(t_{1}-\xi\right)^{\vartheta-1}\right]}{\Gamma(\vartheta)}\left|h_{j}\left(\xi, z_{j}(\xi), z_{j}^{\prime}(\xi)\right)\right| \mathrm{d} \xi \\
& +\int_{t_{1}}^{t_{2}} \frac{\left.\left(t_{2}-\xi\right)\right)^{\vartheta-1}}{\Gamma(\vartheta)}\left|h_{j}\left(\xi, z_{j}(\xi), z_{j}^{\prime}(\xi)\right)\right| \mathrm{d} \xi \\
& +\frac{\left(t_{2}-t_{1}\right)}{|\Delta-1|} \int_{0}^{1} \frac{(1-\xi)^{\vartheta-2}}{\Gamma(\vartheta-1)}\left|h_{j}\left(\xi, z_{j}(\xi), z_{j}^{\prime}(\xi)\right)\right| \mathrm{d} \xi \\
& +\frac{\left(t_{2}-t_{1}\right)}{|\Delta-1|}\left[\int_{0}^{1} \int_{0}^{\xi} \frac{(\xi-m)^{\vartheta-1}}{\Gamma(\vartheta)}\left|h_{j}\left(m, z_{j}(m), z_{j}^{\prime}(m)\right)\right| \mathrm{d} m \mathrm{~d} \xi\right. \\
& +\int_{0}^{1} \int_{0}^{\xi} \frac{(\xi-m)^{\vartheta-2}}{\Gamma(\vartheta-1)}\left|h_{j}\left(m, z_{j}(m), z_{j}^{\prime}(m)\right)\right| \mathrm{d} m \mathrm{~d} \xi \\
& \left.+\int_{0}^{1} \int_{0}^{\xi} \frac{(\xi-m)^{\vartheta-\beta-1}}{\Gamma(\vartheta-\beta)}\left|h_{j}\left(m, z_{j}(m), z_{j}^{\prime}(m)\right)\right| \mathrm{d} m \mathrm{~d} \xi\right] \\
& \leq \frac{\varrho_{j}}{\Gamma(\vartheta+1)}\left[-\left(t_{2}-t_{1}\right)^{\vartheta}+\left(t_{2}-t_{1}\right)^{\vartheta}+t_{2}^{\vartheta}-t_{1}^{\vartheta}\right]+\frac{\varrho_{j}\left(t_{2}-t_{1}\right)}{|\Delta-1| \Gamma(\vartheta)} \\
& +\frac{\varrho_{j}\left(t_{2}-t_{1}\right)}{|\Delta-1|}\left[\frac{1}{\Gamma(\vartheta+2)}+\frac{1}{\Gamma(\vartheta+1)}+\frac{1}{\Gamma(\vartheta-\beta+2)}\right] .
\end{aligned}
$$

The right-side converges to zero (no depending upon $z \in \mathcal{O}$ ) as $t_{1} \rightarrow t_{2}$. Similarly, we have

$$
\left|\left(\mathrm{Y}_{j} z_{j}\right)^{\prime}\left(t_{2}\right)-\left(\mathrm{Y}_{j} z_{j}\right)^{\prime}\left(t_{1}\right)\right| \rightarrow 0,
$$

as $t_{1} \rightarrow t_{2}$. Hence, $\left\|\mathrm{Y} z\left(t_{2}\right)-\mathrm{Y} z\left(t_{1}\right)\right\|_{\mathcal{M}} \rightarrow 0$ as $t_{1} \rightarrow t_{2}$. This gives that $\mathrm{Y}$ is equi-continuous on $\mathcal{M}$. Hence $\mathrm{Y}$ is completely continuous by Arzela-Ascoli theorem. Assume a subset

$$
\Omega:=\left\{\left(z_{1}, z_{2}, \ldots, z_{13}\right) \in \mathcal{M}:\left(z_{1}, z_{2}, \ldots, z_{13}\right)=v Y\left(z_{1}, z_{2}, \ldots, z_{13}\right), v \in(0,1)\right\}
$$

of $\mathcal{M}$. We prove the boundedness of $\Omega$. Let $\left(z_{1}, z_{2}, \ldots, z_{13}\right) \in \Omega$. Then

$$
\left(z_{1}, z_{2}, \ldots, z_{13}\right)=v Y\left(z_{1}, z_{2}, \ldots, z_{13}\right)
$$


and so $z_{j}(t)=v\left(\mathrm{Y}_{j} z_{j}\right)$ for all $t \in[0,1]$ and $j \in \mathbb{N}_{1}^{13}$. Thus,

$$
\begin{aligned}
\left|z_{j}(t)\right| & \leq v\left(\int_{0}^{t} \frac{(t-\xi)^{\vartheta-1}}{\Gamma(\vartheta)}\left|h_{j}\left(\xi, z_{j}(\xi), z_{j}^{\prime}(\xi)\right)\right| \mathrm{d} \xi\right. \\
& +\frac{t+|\Delta|}{|\Delta-1|} \int_{0}^{1} \frac{(1-\xi)^{\vartheta-2}}{\Gamma(\vartheta-1)}\left|h_{j}\left(\xi, z_{j}(\xi), z_{j}^{\prime}(\xi)\right)\right| \mathrm{d} \xi \\
& +\frac{1-t}{|\Delta-1|}\left[\int_{0}^{1} \int_{0}^{\xi} \frac{(\xi-m)^{\vartheta-1}}{\Gamma(\vartheta)}\left|h_{j}\left(m, z_{j}(m), z_{j}^{\prime}(m)\right)\right| \mathrm{d} m \mathrm{~d} \xi\right. \\
& +\int_{0}^{1} \int_{0}^{\xi} \frac{(\xi-m)^{\vartheta-2}}{\Gamma(\vartheta-1)}\left|h_{j}\left(m, z_{j}(m), z_{j}^{\prime}(m)\right)\right| \mathrm{d} m \mathrm{~d} \xi \\
& \left.\left.+\int_{0}^{1} \int_{0}^{\xi} \frac{(\xi-m)^{\vartheta-\beta-1}}{\Gamma(\vartheta-\beta)}\left|h_{j}\left(m, z_{j}(m), z_{j}^{\prime}(m)\right)\right| \mathrm{d} m \mathrm{~d} \xi\right]\right) \\
& \leq v \varrho_{j}\left[\frac{1+|\Delta|}{|\Delta-1| \Gamma(\vartheta)}+\frac{1}{|\Delta-1| \Gamma(\vartheta+2)}+\frac{|\Delta-1|+1}{|\Delta-1| \Gamma(\vartheta+1)}+\frac{1}{|\Delta-1| \Gamma(\vartheta-\beta+2)}\right] \\
& =v \varrho_{j} \mathcal{K}_{0}^{*},
\end{aligned}
$$

and

$$
\left|z_{j}^{\prime}(t)\right| \leq v \varrho_{j}\left[\frac{|\Delta-1|+1}{|\Delta-1| \Gamma(\vartheta)}+\frac{1}{|\Delta-1| \Gamma(\vartheta+2)}+\frac{1}{|\Delta-1| \Gamma(\vartheta-\beta+2)}+\frac{1}{|\Delta-1| \Gamma(\vartheta+1)}\right]=v \varrho_{j} \mathcal{K}_{1}^{*} .
$$

These inequalities follow that

$$
\|z\|_{\mathcal{M}}=\sum_{j=1}^{13}\left\|z_{j}\right\|_{\mathcal{M}_{j}} \leq v \sum_{j=1}^{13} \varrho_{j}\left(\mathcal{K}_{0}^{*}+\mathcal{K}_{1}^{*}\right)<\infty
$$

therefore $\Omega$ is bounded. Utilizing Lemmas 2 and 3, we find out that in $\mathcal{M}, \mathrm{Y}$ possesses a fixed point, which is a solution of (14).

\section{Ulam-Hyers Stability}

Since the concept of stability is so important in determining the solutions to many dynamical systems, due to this importance, we here establish two Ulam-Hyers and the generalized version of stabilities in relation to the fractional multi-dimensional BVP on the methylpropane graph (14). For more information, see [38-43]. In consistence with [41], we define the following notions:

Definition 1 ([41]). The fractional multi-dimensional BVP on the methylpropane graph (14) is Ulam-Hyers stable if $\exists d_{h_{j}}^{*} \in \mathbb{R}_{+}$s.t. $\forall \varepsilon_{j}>0$ and $\forall z_{j}^{*}(t) \in C([0,1], \mathbb{R})$ as a solution function satisfying

$$
\left|{ }^{c} \mathcal{D}_{0}^{\vartheta} z_{j}^{*}(t)-h_{j}\left(t, z_{j}^{*}(t), z_{j}^{*^{\prime}}(t)\right)\right|<\varepsilon_{j},
$$

$\exists z_{j}(t) \in C([0,1], \mathbb{R})$ fulfilling the multi-dimensional BVP on the methylpropane graph (14) with

$$
\left|z_{j}^{*}(t)-z_{j}(t)\right| \leq \varepsilon_{j} d_{h_{j}}^{*}, \quad t \in[0,1]
$$


Definition 2 ([41]). The fractional multi-dimensional BVP on the methylpropane graph (14) is generalized Ulam-Hyers stable if $\exists G_{j} \in C\left(\mathbb{R}^{+}, \mathbb{R}^{+}\right)$with $G_{j}(0)=0$ s.t. $\forall \varepsilon_{j}>0$ and $\forall z_{j}^{*}(t) \in C([0,1], \mathbb{R})$ fulfilling

$$
\left|{ }^{c} \mathcal{D}_{0}^{\vartheta} z_{j}^{*}(t)-h_{j}\left(t, z_{j}^{*}(t), z_{j}^{*^{\prime}}(t)\right)\right|<\varepsilon_{j},
$$

$\exists z_{j}(t) \in C([0,1], \mathbb{R})$ satisfying the multi-dimensional BVP on the methylpropane graph (14) with

$$
\left|z_{j}^{*}(t)-z_{j}(t)\right| \leq G_{j}\left(\varepsilon_{j}\right), \quad t \in[0,1] .
$$

Remark 1. Definition $1 \Rightarrow$ Definition 2.

Remark 2. $z_{j}^{*}(t) \in C([0,1], \mathbb{R})$ is named as a solution for $(16)$ iff $\exists H_{j} \in C([0,1], \mathbb{R})$ depending on $z_{j}^{*}$ s.t.

(1) $\left|H_{j}(t)\right|<\varepsilon_{j}, t \in[0,1]$.

(2) ${ }^{c} \mathcal{D}_{0}^{\vartheta} z_{j}^{*}(t)=h_{j}\left(t, z_{j}^{*}(t), z_{j}^{*^{\prime}}(t)\right)+H_{j}(t), t \in[0,1]$.

We now study the above stabilities for the fractional multi-dimensional BVP on the methylpropane graph (14).

Theorem 3. Assume that the assertion

$$
\left|h_{j}\left(t, z_{j}^{*}(t), z_{j}^{*^{\prime}}(t)\right)-h_{j}\left(t, z_{j}(t), z_{j}^{\prime}(t)\right)\right| \leq \mathcal{L}_{j}^{*}\left(\left|z_{j}^{*}-z_{j}\right|+\left|z_{j}^{*^{\prime}}-z_{j}^{\prime}\right|\right),
$$

for some $\mathcal{L}_{j}^{*}>0$. Then the fractional multi-dimensional BVP on methylpropane graph (14) is Ulam-Hyers stable on $[0,1]$ and is generalized Ulam-Hyers stable if

$$
\mathcal{L}_{j}^{*} \mathcal{K}_{0}^{*}<1, \quad j \in \mathbb{N}_{1}^{13},
$$

for which $\mathcal{K}_{0}^{*}$ is given in (10).

Proof. $\forall \varepsilon_{j}>0$ and $\forall z_{j}^{*} \in C([0,1], \mathbb{R})$ fulfilling

$$
\left|{ }^{c} \mathcal{D}_{0}^{\vartheta} z_{j}^{*}(t)-h_{j}\left(t, z_{j}^{*}(t), z_{j}^{*^{\prime}}(t)\right)\right|<\varepsilon_{j},
$$

one can find $H_{j} \in C([0,1], \mathbb{R})$, which satisfies

$$
{ }^{c} \mathcal{D}_{0}^{\vartheta} z_{j}^{*}(t)=h_{j}\left(t, z_{j}^{*}(t), z_{j}^{*^{\prime}}(t)\right)+H_{j}(t),
$$

with $\left|H_{j}(t)\right| \leq \varepsilon_{j}$. It gives

$$
\begin{aligned}
z_{j}^{*}(t) & =\int_{0}^{t} \frac{(t-\xi)^{\vartheta-1}}{\Gamma(\vartheta)}\left(h_{j}\left(\xi, z_{j}^{*}(\xi), z_{j}^{*^{\prime}}(\xi)\right)+H_{j}(\xi)\right) \mathrm{d} \xi \\
& +\frac{t-\Delta}{\Delta-1} \int_{0}^{1} \frac{(1-\xi)^{\vartheta-2}}{\Gamma(\vartheta-1)}\left(h_{j}\left(\xi, z_{j}^{*}(\xi), z_{j}^{*^{\prime}}(\xi)\right)+H_{j}(\xi)\right) \mathrm{d} \xi \\
& +\frac{1-t}{\Delta-1}\left[\int_{0}^{1} \int_{0}^{\xi} \frac{(\xi-m)^{\vartheta-1}}{\Gamma(\vartheta)}\left(h_{j}\left(m, z_{j}^{*}(m), z_{j}^{*^{\prime}}(m)\right)+H_{j}(m)\right) \mathrm{d} m \mathrm{~d} \xi\right. \\
& +\int_{0}^{1} \int_{0}^{\xi} \frac{(\xi-m)^{\vartheta-2}}{\Gamma(\vartheta-1)}\left(h_{j}\left(m, z_{j}^{*}(m), z_{j}^{*^{\prime}}(m)\right)+H_{j}(m)\right) \mathrm{d} m \mathrm{~d} \xi
\end{aligned}
$$




$$
\left.+\int_{0}^{1} \int_{0}^{\xi} \frac{(\xi-m)^{\vartheta-\beta-1}}{\Gamma(\vartheta-\beta)}\left(h_{j}\left(m, z_{j}^{*}(m), z_{j}^{*^{\prime}}(m)\right)+H_{j}(m)\right) \mathrm{d} m \mathrm{~d} \tilde{\xi}\right] .
$$

Consider $z_{j}(t) \in C(J, \mathbb{R})$ to be the solution of (14), which is

$$
\begin{aligned}
z_{j}(t) & =\int_{0}^{t} \frac{(t-\xi)^{\vartheta-1}}{\Gamma(\vartheta)} h_{j}\left(\xi, z_{j}(\xi), z_{j}^{\prime}(\xi)\right) \mathrm{d} \xi+\frac{t-\Delta}{\Delta-1} \int_{0}^{1} \frac{(1-\xi)^{\vartheta-2}}{\Gamma(\vartheta-1)} h_{j}\left(\xi, z_{j}(\xi), z_{j}^{\prime}(\xi)\right) \mathrm{d} \xi \\
& +\frac{1-t}{\Delta-1}\left[\int_{0}^{1} \int_{0}^{\xi} \frac{(\xi-m)^{\vartheta-1}}{\Gamma(\vartheta)} h_{j}\left(m, z_{j}(m), z_{j}^{\prime}(m)\right) \mathrm{d} m \mathrm{~d} \xi\right. \\
& +\int_{0}^{1} \int_{0}^{\xi} \frac{(\xi-m)^{\vartheta-2}}{\Gamma(\vartheta-1)} h_{j}\left(m, z_{j}(m), z_{j}^{\prime}(m)\right) \mathrm{d} m \mathrm{~d} \xi \\
& \left.+\int_{0}^{1} \int_{0}^{\xi} \frac{(\xi-m)^{\vartheta-\beta-1}}{\Gamma(\vartheta-\beta)} h_{j}\left(m, z_{j}(m), z_{j}^{\prime}(m)\right) \mathrm{d} m \mathrm{~d} \xi\right] .
\end{aligned}
$$

We estimate

$$
\begin{aligned}
& \left|z_{j}^{*}(t)-z_{j}(t)\right| \leq \int_{0}^{t} \frac{(t-\xi)^{\vartheta-1}}{\Gamma(\vartheta)}\left|h_{j}\left(\xi, z_{j}^{*}(\xi), z_{j}^{*^{\prime}}(\xi)\right)-h_{j}\left(\xi, z_{j}(\xi), z_{j}^{\prime}(\xi)\right)\right| \mathrm{d} \xi \\
& +\frac{t+|\Delta|}{|\Delta-1|} \int_{0}^{1} \frac{(1-\xi)^{\vartheta-2}}{\Gamma(\vartheta-1)}\left|h_{j}\left(\xi, z_{j}^{*}(\xi), z_{j}^{*^{\prime}}(\xi)\right)-h_{j}\left(\xi, z_{j}(\xi), z_{j}^{\prime}(\xi)\right)\right| \mathrm{d} \xi \\
& +\frac{1-t}{|\Delta-1|}\left[\int_{0}^{1} \int_{0}^{\xi} \frac{(\xi-m)^{\vartheta-1}}{\Gamma(\vartheta)}\left|h_{j}\left(m, z_{j}^{*}(m), z_{j}^{*^{\prime}}(m)\right)-h_{j}\left(m, z_{j}(m), z_{j}^{\prime}(m)\right)\right| \mathrm{d} m \mathrm{~d} \xi\right. \\
& +\int_{0}^{1} \int_{0}^{\xi} \frac{(\xi-m)^{\vartheta-2}}{\Gamma(\vartheta-1)}\left|h_{j}\left(m, z_{j}^{*}(m), z_{j}^{*^{\prime}}(m)\right)-h_{j}\left(m, z_{j}(m), z_{j}^{\prime}(m)\right)\right| \mathrm{d} m \mathrm{~d} \xi \\
& \left.+\int_{0}^{1} \int_{0}^{\xi} \frac{(\xi-m)^{\vartheta-\beta-1}}{\Gamma(\vartheta-\beta)}\left|h_{j}\left(m, z_{j}^{*}(m), z_{j}^{*^{\prime}}(m)\right)-h_{j}\left(m, z_{j}(m), z_{j}^{\prime}(m)\right)\right| \mathrm{d} m \mathrm{~d} \xi\right] \\
& +\int_{0}^{t} \frac{(t-\xi)^{\vartheta-1}}{\Gamma(\vartheta)}\left|H_{j}(\xi)\right| \mathrm{d} \xi+\frac{t+|\Delta|}{|\Delta-1|} \int_{0}^{1} \frac{(1-\xi)^{\vartheta-2}}{\Gamma(\vartheta-1)}\left|H_{j}(\xi)\right| \mathrm{d} \xi \\
& +\frac{1-t}{|\Delta-1|}\left[\int_{0}^{1} \int_{0}^{\xi} \frac{(\xi-m)^{\vartheta-1}}{\Gamma(\vartheta)}\left|H_{j}(m)\right| \mathrm{d} m \mathrm{~d} \xi+\int_{0}^{1} \int_{0}^{\xi} \frac{(\xi-m)^{\vartheta-2}}{\Gamma(\vartheta-1)}\left|H_{j}(m)\right| \mathrm{d} m \mathrm{~d} \xi\right. \\
& \left.+\int_{0}^{1} \int_{0}^{\xi} \frac{(\xi-m)^{\vartheta-\beta-1}}{\Gamma(\vartheta-\beta)}\left|H_{j}(m)\right| \mathrm{d} m \mathrm{~d} \xi\right] \\
& \leq \mathcal{L}_{j}^{*}\left\|z_{j}^{*}-z_{j}\right\|\left[\frac{1+|\Delta|}{|\Delta-1| \Gamma(\vartheta)}+\frac{1}{|\Delta-1| \Gamma(\vartheta+2)}\right. \\
& \left.+\frac{|\Delta-1|+1}{|\Delta-1| \Gamma(\vartheta+1)}+\frac{1}{|\Delta-1| \Gamma(\vartheta-\beta+2)}\right]
\end{aligned}
$$




$$
\begin{aligned}
& +\varepsilon_{j}\left[\frac{1+|\Delta|}{|\Delta-1| \Gamma(\vartheta)}+\frac{1}{|\Delta-1| \Gamma(\vartheta+2)}+\frac{|\Delta-1|+1}{|\Delta-1| \Gamma(\vartheta+1)}+\frac{1}{|\Delta-1| \Gamma(\vartheta-\beta+2)}\right] \\
& =\varepsilon_{j} \mathcal{K}_{0}^{*}+\mathcal{K}_{0}^{*} \mathcal{L}_{j}^{*}\left\|z_{j}^{*}-z_{j}\right\| .
\end{aligned}
$$

Hence

$$
\left\|z_{j}^{*}-z_{j}\right\| \leq \varepsilon_{j} \mathcal{K}_{0}^{*}+\mathcal{K}_{0}^{*} \mathcal{L}_{j}^{*}\left\|z_{j}^{*}-z_{j}\right\|,
$$

in which $\mathcal{K}_{0}^{*}$ is known in (10). From above, we obtain that

$$
\left\|z_{j}^{*}-z_{j}\right\| \leq \frac{\varepsilon_{j} \mathcal{K}_{0}^{*}}{1-\mathcal{K}_{0}^{*} \mathcal{L}_{j}^{*}}
$$

and thus by taking $d_{h_{j}}^{*}=\frac{\mathcal{K}_{0}^{*}}{1-\mathcal{K}_{0}^{*} \mathcal{L}_{j}^{*}}$, we find that the multi-dimensional methylpropane FBVP (14) is Ulam-Hyers stable $\left(\mathcal{K}_{0}^{*} \mathcal{L}_{j}^{*}<1\right)$. Further, for $G_{j}\left(\varepsilon_{j}\right)=\frac{\varepsilon_{j} \mathcal{K}_{0}^{*}}{1-\mathcal{K}_{0}^{*} \mathcal{L}_{j}^{*}}$ with $G_{j}(0)=$ 0 , we have the generalized Ulam-Hyers stability for the mentioned multi-dimensional methylpropane FBVP (14).

Example 2. Consider the multi-term multi-dimensional DEs

$$
\left\{\begin{array}{l}
{ }^{c} \mathcal{D}_{0}^{1.38} z_{1}(t)=h_{1}\left(t, z_{1}(t), z_{1}^{\prime}(t)\right), \\
{ }^{c} \mathcal{D}_{0}^{1.38} z_{2}(t)=h_{2}\left(t, z_{2}(t), z_{2}^{\prime}(t)\right), \\
{ }^{c} \mathcal{D}_{0}^{1.38} z_{3}(t)=h_{3}\left(t, z_{3}(t), z_{3}^{\prime}(t)\right), \\
{ }^{c} \mathcal{D}_{0}^{1.38} z_{4}(t)=h_{4}\left(t, z_{4}(t), z_{4}^{\prime}(t)\right),
\end{array}\right.
$$

under the integral $B C s$

$$
\left\{\begin{array}{l}
z_{j}(0)+{ }^{c} \mathcal{D}_{0}^{0.47} z_{j}(0)+z_{j}^{\prime}(1)=0, \quad(j=1,2,3,4) \\
\int_{0}^{1}\left[z_{j}(\xi)+{ }^{c} \mathcal{D}_{0}^{0.47} z_{j}(\xi)+z_{j}^{\prime}(\xi)\right] \mathrm{d} \xi=0,
\end{array}\right.
$$

where $\vartheta=1.38, \beta=0.47, n=4$, and ${ }^{c} \mathcal{D}_{0}^{1.38}$ and ${ }^{c} \mathcal{D}_{0}^{0.47}$ are derivatives of order $\gamma \in\{1.38,0.47\}$ of the Caputo type. Set $h_{j} \in C\left([0,1] \times \mathbb{R}^{3}, \mathbb{R}\right),(j=1, \ldots, 4)$ by

$$
\left\{\begin{array}{l}
h_{1}(t, z(t), \ell(t))=\frac{|\sin z(t)|}{8}+\frac{|\ell(t)|}{8}+e^{t}, \\
h_{2}(t, z(t), \ell(t))=\frac{0.1 e|z(t)|}{5+5|z(t)|}+\frac{e|\sin \ell(t)|}{50(1+|\sin \ell(t)|)}+2 \sin (t), \\
h_{3}(t, z(t), \ell(t))=2+\frac{1}{700}|\arctan z(t)|+\frac{|\ell(t)|}{700+700|\ell(t)|}, \\
h_{4}(t, z(t), \ell(t))=\frac{e}{80}|z(t)|+0.0125 e|\ell(t)|-21 t .
\end{array}\right.
$$

By the mean value theorem, we know that $|\sin a-\sin b| \leq|a-b|$ and $\mid \arctan a-$ $\arctan b|\leq| a-b \mid$ for all $a, b \in \mathbb{R}$. Thus, for $z_{1}, z_{2}, \ell_{1}, \ell_{2} \in \mathbb{R}$, we estimate

$$
\left|h_{1}\left(t, z_{1}(t), z_{2}(t)\right)-h_{1}\left(t, \ell_{1}(t), \ell_{2}(t)\right)\right|
$$




$$
\begin{aligned}
& \leq \frac{1}{8}\left(\left|\sin z_{1}(t)-\sin \ell_{1}(t)\right|+\left|z_{2}(t)-\ell_{2}(t)\right|\right) \\
& \leq \frac{1}{8}\left(\left|z_{1}(t)-\ell_{1}(t)\right|+\left|z_{2}(t)-\ell_{2}(t)\right|\right), \\
& \mid h_{2}\left(t, z_{1}(t), z_{2}(t)\right)- h_{2}\left(t, \ell_{1}(t), \ell_{2}(t)\right) \mid \\
& \leq \frac{0.1 e}{5}\left(\left|z_{1}(t)-\ell_{1}(t)\right|+\left|\sin z_{2}(t)-\sin \ell_{2}(t)\right|\right) \\
& \leq \frac{0.1 e}{5}\left(\left|z_{1}(t)-\ell_{1}(t)\right|+\left|z_{2}(t)-\ell_{2}(t)\right|\right), \\
&\left|h_{3}\left(t, z_{1}(t), z_{2}(t)\right)-h_{3}\left(t, \ell_{1}(t), \ell_{2}(t)\right)\right| \\
& \leq \frac{1}{700}\left(\left|\arctan z_{1}(t)-\arctan \ell_{1}(t)\right|+\left|z_{2}(t)-\ell_{2}(t)\right|\right) \\
& \leq \frac{1}{700}\left(\left|z_{1}(t)-\ell_{1}(t)\right|+\left|z_{2}(t)-\ell_{2}(t)\right|\right),
\end{aligned}
$$

and

$$
\begin{aligned}
\mid h_{4}\left(t, z_{1}(t), z_{2}(t)\right) & -h_{4}\left(t, \ell_{1}(t), \ell_{2}(t)\right) \mid \\
& \leq \frac{e}{80}\left(\left|z_{1}(t)-\ell_{1}(t)\right|+\left|z_{2}(t)-\ell_{2}(t)\right|\right) \\
& \leq \frac{e}{80}\left(\left|z_{1}(t)-\ell_{1}(t)\right|+\left|z_{2}(t)-\ell_{2}(t)\right|\right) .
\end{aligned}
$$

Hence, $\mathcal{L}_{1}^{*}=\frac{1}{8}, \mathcal{L}_{2}^{*}=\frac{0.1 e}{5}, \mathcal{L}_{3}^{*}=\frac{1}{700}$, and $\mathcal{L}_{4}^{*}=\frac{e}{80}$. According to the obtained values, we obtain $\Delta \simeq 2.2364$ and $\mathcal{K}_{0}^{*} \simeq 5.1371$. Since,

$$
\begin{aligned}
& \mathcal{L}_{1}^{*} \mathcal{K}_{0}^{*} \simeq 0.64213<1, \quad \mathcal{L}_{2}^{*} \mathcal{K}_{0}^{*} \simeq 0.27843<1, \\
& \mathcal{L}_{3}^{*} \mathcal{K}_{0}^{*} \simeq 0.007338<1, \quad \mathcal{L}_{4}^{*} \mathcal{K}_{0}^{*} \simeq 0.17401<1 .
\end{aligned}
$$

Then by taking the constants

$$
\begin{aligned}
& d_{h_{1}}^{*}=\frac{\mathcal{K}_{0}^{*}}{1-\mathcal{K}_{0}^{*} \mathcal{L}_{1}^{*}} \simeq 14.354653, \quad d_{h_{2}}^{*}=\frac{\mathcal{K}_{0}^{*}}{1-\mathcal{K}_{0}^{*} \mathcal{L}_{2}^{*}} \simeq 7.119337, \\
& d_{h_{3}}^{*}=\frac{\mathcal{K}_{0}^{*}}{1-\mathcal{K}_{0}^{*} \mathcal{L}_{3}^{*}} \simeq 5.175085, \quad d_{h_{4}}^{*}=\frac{\mathcal{K}_{0}^{*}}{1-\mathcal{K}_{0}^{*} \mathcal{L}_{4}^{*}} \simeq 6.219324,
\end{aligned}
$$

the conclusion of Theorem 3 confirms that the fractional system of BVPs (17) and (18) is Ulam-Hyers stable. Moreover, by defining the functions

$$
\begin{aligned}
& G_{1}\left(\varepsilon_{1}\right)=\frac{\varepsilon_{1} \mathcal{K}_{0}^{*}}{1-\mathcal{K}_{0}^{*} \mathcal{L}_{1}^{*}} \simeq 14.354653 \varepsilon_{1}, \quad G_{2}\left(\varepsilon_{2}\right)=\frac{\varepsilon_{2} \mathcal{K}_{0}^{*}}{1-\mathcal{K}_{0}^{*} \mathcal{L}_{2}^{*}} \simeq 7.119337 \varepsilon_{2}, \\
& G_{3}\left(\varepsilon_{3}\right)=\frac{\varepsilon_{3} \mathcal{K}_{0}^{*}}{1-\mathcal{K}_{0}^{*} \mathcal{L}_{3}^{*}} \simeq 5.175085 \varepsilon_{3}, \quad G_{4}\left(\varepsilon_{4}\right)=\frac{\varepsilon_{4} \mathcal{K}_{0}^{*}}{1-\mathcal{K}_{0}^{*} \mathcal{L}_{4}^{*}} \simeq 6.219324 \varepsilon_{4},
\end{aligned}
$$


so that $G_{j}(0)=0,(j=1,2,3,4)$, we see that the conclusion of Theorem 3 implies that the fractional system of BVPs (17)-(18) is the generalized Ulam-Hyers stable.

\title{
5. Conclusions
}

In this study, we extended the technique applied in $[28,29]$ to the non-star methylpropane graph and studied a new family of multi-term multi-dimensional FBVPs on every edge of the graph by introducing a labeling method for vertices. We discussed the existence of solutions for aforesaid FBVPs with the aid of Krasnoselskii and Schaefer fixed point theorems. Moreover, the stability analysis in the sense of Ulam-Hyers and generalized Ulam-Hyers was investigated. In addition, we prepared an abstract example to explain one of our results. In view of the importance of differential equations in chemical graph theory, we designed our FBVPs (2) on the molecular graph representation of methylpropane as an example of a non-star graph. This research is of pivotal nature for fractional mathematical modeling on different chemical compounds of molecules in the framework of complicated non-star graphs. Our suggested FBVPs are applicable on a vast range of graph structures, especially digraphs, which are usually utilized in medical technologies in relation to protein networks. In our future projects, we plan to study more problems on the graph representation of different molecular structures by using analytic and numerical methods.

Author Contributions: Conceptualization, S.R. and S.E.; Formal analysis, S.R., C.T.D., A.H., S.E., R.G and B.A.; Funding acquisition, R.G.; Methodology, S.R., C.T.D., A.H., S.E., R.G. and B.A.; Software, S.E. All authors have read and agreed to the published version of the manuscript.

Funding: This research received no external funding.

Institutional Review Board Statement: Not applicable.

Informed Consent Statement: Not applicable.

Data Availability Statement: Data sharing not applicable to this article as no datasets were generated or analyzed during the current study.

Acknowledgments: The first and fourth authors would like to thank Azarbaijan Shahid Madani University. Also, the authors would like to sincerely thank the reviewers for their constructive comments to improve the quality of the paper.

Conflicts of Interest: The authors declare no conflict of interest.

\author{
Abbreviations \\ The following abbreviations are used in this manuscript: \\ FBVP Fractional Boundary Value Problem, \\ ODEs Ordinary Differential Equations, \\ BCs Boundary Conditions, \\ RL Riemann-Liouville, \\ DEs Differential Equations.
}

\section{References}

1. Adjabi, Y.; Samei, M.E.; Matar, M.M.; Alzabut, J. Langevin differential equation in frame of ordinary and Hadamard fractional derivatives under three point boundary conditions. AIMS Math. 2021, 6, 2796-2843. [CrossRef]

2. Rezapour, S.; Etemad, S.; Mohammadi, H. A mathematical analysis of a system of Caputo-Fabrizio fractional differential equations for the anthrax disease model in animals. Adv. Differ. Equ. 2020, 2020, 481. [CrossRef]

3. Deressa, C.T.; Etemad, S.; Rezapour, S. On a new four-dimensional model of memristor-based chaotic circuit in the context of nonsingular Atangana-Baleanu-Caputo operators. Adv. Differ. Equ. 2021, 2021, 444. [CrossRef]

4. Nagajothi, N.; Sadhasivam, V.; Bazighifan, O.; El-Nabulsi, R.A. Existence of the class of nonlinear hybrid fractional Langevin quantum differential equation with Dirichlet boundary conditions. Fractal Fract. 2021, 5, 156. [CrossRef]

5. Khan, H.; Tunc, C.; Chen, W.; Khan, A. Existence theorems and Hyers-Ulam stability for a class of hybrid fractional differential equations with $p$-Laplacial operator. J. Appl. Anal. Comput. 2018, 8, 1211-1226.

6. Deressa, C.T.; Etemad, S.; Kaabar, M.K.A.; Rezapour, S. Qualitative analysis of a hyperchaotic Lorenz-Stenflo mathematical model via the Caputo fractional operator. J. Funct. Spaces 2022, 2022, 4975104. [CrossRef] 
7. Salem, A.; Alghamdi, B. Multi-strip and multi-point boundary conditions for fractional Langevin equation. Fractal Fract. 2020, 4, 18. [CrossRef]

8. Alzabut, J.; Selvam, G.M.; El-Nabulsi, R.A.; Vignesh, D.; Samei, M.E. Asymptotic stability of nonlinear discrete fractional pantograph equations with non-local initial conditions. Symmetry 2021, 13, 473. [CrossRef]

9. Nieto, J.J.; Pimentel, J. Positive solutions of a fractional thermostat model. Bound. Value Probl. 2013, 2013, 5. [CrossRef]

10. Mohammadi, H.; Kumar, S.; Rezapour, S.; Etemad, S. A theoretical study of the Caputo-Fabrizio fractional modeling for hearing loss due to Mumps virus with optimal control. Chaos Solitons Fractals 2021, 144, 110668. [CrossRef]

11. Afshari, H.; Kalantari, S.; Karapinar, E. Solution of fractional differential equations via coupled fixed point. Electron. J. Differe. Equ. 2015, 2015, 286.

12. Rezapour, S.; Tellab, B.; Deressa, C.T.; Etemad, S.; Nonlaopon, K. H-U-type stability and numerical solutions for a nonlinear model of the coupled systems of Navier BVPs via the generalized differential transform method. Fractal Fract. 2021, 5(4), 166. [CrossRef]

13. Baleanu, D.; Etemad, S.; Pourrazi, S.; Rezapour, S. On the new fractional hybrid boundary value problems with three-point integral hybrid conditions. Adv. Differ. Equ. 2019, 2019, 473. [CrossRef]

14. Bachir, F.S.; Abbas, S.; Benbachir, M.; Benchohra, M. Hilfer-Hadamard fractional differential equations; Existence and attractivity Adv. Theory Nonlinear Anal. Appl. 2021, 5, 49-57.

15. Rezapour, S.; Ntouyas, S.K.; Iqbal, M.Q.; Hussain, A.; Etemad, S.; Tariboon, J. An analytical survey on the solutions of the generalized double-order $\phi$-integro-differential equation. J. Funct. Spaces 2021, 2021, 6667757.

16. Abdeljawad, T.; Agarwal, R.P.; Karapinar, E.; Kumari. Solutions of the nonlinear integral equation and fractional differential equation using the technique of a fixed point with a numerical experiment in extended b-metric space. Symmetry 2019, 11, 686. [CrossRef]

17. Amara, A.; Etemad, S.; Rezapour, S. Topological degree theory and Caputo-Hadamard fractional boundary value problems. Adv. Differ. Equ. 2020, 2020, 369. [CrossRef]

18. Alqahtani, B.; Aydi, H.; Karapinar, E.; Rakocevic, V. A solution for Volterra fractional integral equations by hybrid contractions Mathematics 2019, 7, 694. [CrossRef]

19. Etemad, S.; Ntouyas, S.K.; Ahmad, B. Existence theory for a fractional $q$-integro-difference equation with $q$-integral boundary conditions of different orders. Mathematics 2019, 7, 659. [CrossRef]

20. Shah, K.; Khan, R.A. Iterative scheme for a coupled system of fractional-order differential equations with three-point boundary conditions. Math. Methods Appl. Sci. 2018, 41, 1047-1053. [CrossRef]

21. Etemad, S.; Rezapour, S.; Samei, M.E. $\alpha-\psi$-contractions and solutions of a $q$-fractional differential inclusion with three-point boundary value conditions via computational results. Adv. Differ. Equ. 2020, 2020, 218. [CrossRef]

22. Wongcharoen, A.; Ntouyas, S. K.; Tariboon, J. Nonlocal boundary value problems for Hilfer type pantograph fractional differential equations and inclusions. Adv. Differ. Equ. 2020, 2020, 279. [CrossRef]

23. Thaiprayoon, C.; Sudsutad, W.; Alzabut, J.; Etemad, S.; Rezapour, S. On the qualitative analysis of the fractional boundary value problem describing thermostat control model via $\psi$-Hilfer fractional operator. Adv. Differ. Equ. 2021, 2021. 201. [CrossRef]

24. Lumer, G. Connecting of local operators and evolution equations on a network. Lect. Notes Math. 1980, 787, 219-234.

25. Nicaise, S. Some results on spectral theory over networks applied to nerve impulses transmission. Lect. Notes Math. 1985, 1771, 532-541.

26. Zavgorodnii, M.G.; Pokornyi, Y.V. On the spectrum of second-order boundary value problems on spatial networks. Usp. Mat. Nauk. 1989, 44, 220-221.

27. Gordeziani, D.G.; Kupreishvli, M.; Meladze, H.V.; Davitashvili, T.D. On the solution of boundary value problem for differential equations given in graphs. Appl. Math. Lett. 2008, 13, 80-91.

28. Graef, J.R.; Kong, L.J.; Wang, M. Existence and uniqueness of solutions for a fractional boundary value problem on a graph. Fract. Calc. Appl. Anal. 2014, 17, 499-510. [CrossRef]

29. Mehandiratta, V.; Mehra, M.; Leugering, G. Existence and uniqueness results for a nonlinear Caputo fractional boundary value problem on a star graph. J. Math. Anal. Appl. 2019, 477, 1243-1264. [CrossRef]

30. Baleanu, D.; Etemad, S.; Mohammadi, H.; Rezapour, S. A novel modeling of boundary value problems on the glucose graph Commun. Nonlinear Sci. Numer. Simulat. 2021, 100, 105844. [CrossRef]

31. Turab, A.; Mitrovic, Z.D.; Savic, A. Existence of solutions for a class of nonlinear boundary value problems on the hexasilinane graph. Adv. Differ. Equ. 2021, 2021, 494. [CrossRef]

32. Turab, A.; Sintunavarat, W. The novel existence results of solutions for a nonlinear fractional boundary value problem on the ethane graph. Alex. Eng. J. 2021, 60, 5365-5374. [CrossRef]

33. Etemad, S.; Rezapour, S. On the existence of solutions for fractional boundary value problems on the ethane graph. Adv. Differ. Equ. 2020, 2020, 276. [CrossRef]

34. Podlubny, I. Fractional Differential Equations; Academic Press: Cambridge, MA, USA, 1999.

35. Samko, S.G.; Kilbas, A.A.; Marichev, O. Fractional Integrals and Derivatives: Theory and Applications; Gordon and Breach: New York, NY, USA, 1993.

36. Miller, K.S.; Ross, B. An Introduction to the Fractional Calculus and Fractional Differential Equations; John Wiley Sons: Hoboken, NJ, USA, 1993. 
37. Smart, D.R. Fixed Point Theorems; Cambridge University Press: Cambridge, UK, 1980.

38. Matar, M.M.; Abu Skhail, E.S. On stability of nonautonomous perturbed semilinear fractional differential systems of order $\alpha \in(1,2) . J$. Math. 2018, 2018, 1723481. [CrossRef]

39. Ameen, R.; Jarad, F.; Abdeljawad, T. Ulam stability for delay fractional differential equations with a generalized Caputo derivative. Filomat 2018, 32, 5265-5274. [CrossRef]

40. Ben-Chikh, S.; Amara, A.; Etemad, S.; Rezapour, S. On Hyers-Ulam stability of a multi-order boundary value problems via Riemann-Liouville derivatives and integrals. Adv. Differ. Equ. 2020, 2020, 547. [CrossRef]

41. Rus, I.A. Ulam stabilities of ordinary differential equations in a Banach space. Carpathian J. Math. 2010, 26, 103-107.

42. Wang, J.R.; Li, X. A uniform method to Ulam-Hyers stability for some linear fractional equations. Mediterr. J. Math. 2016, 13, 625-635. [CrossRef]

43. Wang, J.R.; Feckan, M.; Zhou, Y. Ulam's type stability of impulsive ordinary differential equations. J. Math. Anal. Appl. 2012, 395, 258-264. [CrossRef] 